Vol. $7, n^{\circ} 1 \mid 2003$

Varia

\title{
La prostitution à Bruxelles pendant la Grande Guerre : contrôle et pratique
}

The Herman Diedericks Prize Essay for 2002 / Lauréat du prix Herman Diedericks 2002

\section{Benoit Majerus}

\section{OpenEdition}

\section{Journals}

Édition électronique

URL : https://journals.openedition.org/chs/603

DOI : $10.4000 /$ chs. 603

ISSN : 1663-4837

Éditeur

Librairie Droz

\section{Édition imprimée}

Date de publication : 1 juillet 2003

Pagination : $5-42$

ISBN : 2-600-00865-9

ISSN : 1422-0857

Référence électronique

Benoit Majerus, «La prostitution à Bruxelles pendant la Grande Guerre : contrôle et pratique », Crime, Histoire \& Sociétés / Crime, History \& Societies [En ligne], Vol. 7, $n^{\circ} 1$ | 2003, mis en ligne le 25 février 2009, consulté le 22 mars 2022. URL : http://journals.openedition.org/chs/603 ; DOI : https://doi.org/ $10.4000 /$ chs. 603

Ce document a été généré automatiquement le 22 mars 2022

(C) Droz 


\section{La prostitution à Bruxelles pendant la Grande Guerre : contrôle et pratique $^{1}$}

The Herman Diedericks Prize Essay for 2002 / Lauréat du prix Herman

Diedericks 2002

\section{Benoit Majerus}

1 Dans l'obscurité, six femmes éclairées, lèvres rouges et chapeaux à plumes sur la tête. Des seins lourds, des culs qui attirent l'attention et des vêtements qui dévoilent plus qu'ils ne cachent. La scène ne laisse planer aucun doute: ce sont des prostituées qui font le trottoir. Les six filles soumises ne sont pas particulièrement attirantes : elles essaient de remédier à leur manque de charme par un 'déguisement' excessif. Si ce groupe de femmes occupe la majeure partie de l'œuvre à droite, on observe de l'autre côté de la rue qui coupe la gravure en deux, un personnage différent. Ses traits sont difficilement déchiffrables; l'homme se trouve dans l'ombre. C'est un soldat. Seule la direction des yeux est parlante : il lorgne vers une des prostituées².

2 Cette gravure a été réalisée en 1924 par le peintre allemand Otto Dix (1891-1969) et porte comme titre 'Soldat du Front à Bruxelles'. Comme beaucoup d'artistes de son pays, Dix part en août 1914 comme volontaire à la guerre pour vivre d'une manière immédiate (unmittelbar) ce conflit qui lui semble essentiel pour son cheminement artistique. Contrairement à Macke qui meurt sur le front ou Beckmann qui y subit un effondrement nerveux, Dix y restera jusqu'au moment où la révolution de novembre 1918 met fin au combat. La plupart du temps, il est engagé sur différents champs de bataille. On peut supposer qu'il est aussi passé par Bruxelles puisqu'il est stationné en automne 1916 et en juillet 1917 près de Langemarck et d'Ypres. Comme on le verra plus loin, Bruxelles constitue un lieu privilégié pour les soldats stationnés sur le front de l'ouest qui y passent leurs brefs congés, le plus connu étant sans doute Adolf Hitler. D'après les témoignages de l'époque, celui-ci sera davantage marqué par l'architecture de Poelaert que par la vie nocturne de Bruxelles. 
3 Il n'est guère étonnant qu'Otto Dix ait choisi de représenter cette scène. Outre les portraits et les paysages, la vie érotique constitue un de ses sujets favoris. D'autres contemporains, comme par exemple George Grosz, choisissent le même thème. Dans les 98 gravures qu'il fait paraître en 1924 dans le cycle 'Der Krieg', seules quatre œuvres abordent la thématique de l'érotisme. Toutes traitent de la prostitution à l'arrière du front. La reproduction en question est la plus explicite sur l'importance de l'amour vénal pendant le conflit. Pour Dix qui veut montrer les choses comme elles sont vraiment $^{3}$, la prostitution semble être le seul loisir dont dispose le soldat pendant les courts moments où il peut s'éloigner du front. La gravure réunit tous les éléments de la Nouvelle Objectivité : description sans ménagement de la réalité à travers le regard social et critique de l'artiste, représentation non pas photographique, mais condensée, caricaturée de la réalité. L'art ne sert pas à créer des fictions, à servir une beauté idéale, mais à souligner d'une manière réaliste le monde. Un monde gris, triste, socialement injuste. Dans la gravure en question, un brin d'ironie confère cependant un caractère moins pesant au tableau.

Otto Dix n'est pas le seul soldat-artiste pour lequel Bruxelles devient le symbole d'une certaine idée de l'Etappenstadt comme lieu de débauche (sans que cela ait nécessairement une connotation négative). La prostitution semble, dans cette conception, constituer un des éléments-clés de l'expérience de l'Arrière.

Cette représentation artistique est née d'une réalité sociale: une demande de prostituées renforcée par la présence d'une armée d'occupation. La problématique est d'ailleurs encore d'actualité aujourd'hui comme l'a montré le récent débat autour des soldats allemands au Kosovo. Jusqu'il y a peu, l'historiographie s'est peu intéressée à ce phénomène. Récemment un ouvrage a permis de déblayer le terrain. Insa Meinen s'est livré à une étude détaillée de l'attitude de l'armée allemande face à la prostitution en France occupée pendant la Deuxième Guerre mondiale. Cet article tente d'analyser les stratégies mises en place à Bruxelles par l'occupant pour contrôler la prostitution qu'il considère comme une menace non-négligeable ainsi que de décrire le monde prostitutionnel pendant ces quatre années. Pour la Première Guerre mondiale, les prostituées sont en quelque sorte doublement des "Oublié[e]s de la Grande Guerre»(Becker) : en tant que membres des populations occupées et en tant que marginales de ces sociétés ${ }^{4}$.

\section{La prostitution à Bruxelles pendant le dix-neuvième siècle}

6 Depuis l'ouvrage pionnier d'Alain Corbin consacré aux 'filles de noce', le phénomène prostitutionnel a attiré l'attention des historiens européens. Ils y voient un élément catalyseur qui permet de confronter des éléments d'histoire politique, sociale et culturelle, ou pour se situer sur un plan plus concret, d'histoire des femmes, du droit, de la médecine et du maintien de l'ordre. Le discours et la pratique à propos de la prostitution révèlent souvent les conceptions sociétales d'une élite, les peurs que celleci peut éprouver. En Belgique, le sujet a été partiellement balisé pour le XIX ${ }^{\mathrm{e}}$ siècle, même si la plupart des recherches se limitent à la ville de Bruxelles 5 . La prostituée en tant que telle reste la grande absente du débat historiographique. 
7 La loi communale du 30 mars 1836 avait attribué aux seules communes la responsabilité de la régulation de la prostitution : «Au collège des bourgmestre et échevins appartient la surveillance des personnes et des lieux notoirement livrés à la débauche. Il prend à cet effet les mesures propres à assurer la sûreté, la moralité et la tranquillité publiques. Le conseil fait à ce sujet tels règlements qu'il juge utiles et nécessaires " ${ }^{6}$. À Bruxelles, un premier règlement entre en vigueur en 1844. Il est fortement influencé par la pensée du théoricien du réglementarisme, Parent-Duchâtelet. Ce médecin a effectué pendant huit ans des recherches sur la prostitution en France; ses travaux, inspirés par le positivisme, ont constitué pour longtemps la Bible en la matière. Pour ParentDuchâtelet «les prostituées sont aussi inévitables, dans une agglomération d'hommes que les égouts, les voiries et les dépôts d'immondices "; il est donc inutile de les interdire. Si la prostitution doit être tolérée, il faut la contrôler pour minimiser les risques. Le danger qui émane des prostituées se décline le long de deux axes: la contagion biologique (c'est-à-dire les maladies vénériennes comme la syphilis) et la contagion morale. Ce dernier danger est encore renforcé par une particularité de la profession, à savoir son caractère temporaire. Les anciennes prostituées réintègrent le plus souvent la société et risquent donc d'y répandre ces vices. Trois principes permettent d'en réduire les effets néfastes : 1) créer un milieu clos qui contient ces activités; 2) cet espace devra être contrôlé par l'administration; 3) le monde prostitutionnel sera hiérarchisé. Ce programme d'apparence scientifique est influencé par la grande ambition des Lumières : cataloguer le savoir et ainsi le maitriser7.

8 Le règlement bruxellois de 1844 sera repris par d'autres villes en Belgique et sera considéré dans le monde scientifique européen comme exemplaire. Il se caractérise par deux mots-clés apparemment contradictoires: transparence et discrétion. Transparence $\mathrm{du}$ monde prostitutionnel pour les services responsables de la surveillance: la vie de la prostituée est étroitement surveillée et les maisons de tolérance doivent toujours être accessibles à la police et aux médecins. Discrétion dans l'espace urbain : la fille publique n'a pas le droit d'attirer trop visiblement le regard des clients potentiels et les 'maisons de débauche' sont obligées de se montrer discrètes.

Mais par son côté rigide, le règlement de 1844 est incapable de suivre les changements qui bouleversent la société au XIX siècle. Pour Alain Corbin, la destruction des anciens quartiers abritant traditionnellement les prostituées, les problèmes de renouvellement du personnel, l'éveil de l'opinion publique et surtout l'évolution de la demande transforment la pratique prostitutionnelle. On assiste à un déclin de la prostitution close, favorisée par l'administration communale car facilement contrôlable, en faveur de la prostitution éparse, beaucoup moins saisissable. Le commissaire en chef de la police de Bruxelles, Lenaers, propose en 1877 un règlement plus libéral par lequel il espère pouvoir faire sortir une partie du milieu prostitutionnel de la clandestinité pour le soumettre ainsi à un nouveau contrôle. D'après Huberty, deux conclusions s'imposent après ce changement de cap : échec de l'hyperréglementation; l'argument sanitaire est partiellement supplanté par l'argument moral'.

Pendant que les édiles de la capitale continuent à appréhender la prostitution selon le paradigme du réglementarisme, un autre courant de pensée qui propose une véritable révolution copernicienne, commence à prendre forme d'abord en Angleterre, puis sur le continent. Le mouvement abolitionniste propose de supprimer toute réglementation parce qu'elle ne produit pas les résultats escomptés et pousse les prostituées dans l'illégalité. Pour les abolitionnistes prohibitionnistes, la prostitution n'est pas un mal 
nécessaire qu'il faut contrôler, mais un mal qu'il faut éradiquer complètement. Un autre courant, plus libéral, s'oppose au réglementarisme au nom de la liberté des personnes à disposer de leur corps. Les deux camps s'élèvent contre l'arbitraire et la corruption de la police des mœurs 9 . Un des exemples les plus médiatisés pour soutenir cette cause sera le scandale de la traite des blanches qui éclate en 1879 à Bruxelles et qui se termine en 1881 après de multiples rebondissements avec la démission du commissaire en chef. Grâce à la pression des mouvements abolitionnistes et de la presse, un trafic de jeunes filles anglaises mineures a été révélé au grand public.

11 À la veille de la Première Guerre mondiale, la prostitution à Bruxelles reste donc soumise à des règles qui n'ont plus vraiment changé depuis presque quarante ans et qui sont encore marquées par l'ancien réglementarisme. En 1900, le projet du ministre de la Justice Lejeune de criminaliser la prostitution et de confier le contrôle de celle-ci à l'État se heurte à l'autonomie communale et est refusé. À en croire Huberty et Keunings, le milieu des maisons closes se caractérise par son manque de dynamisme. En 1914, seuls six bordels tolérés avec 38 pensionnaires subsistent dans la capitale ${ }^{10}$. Un rapport de police en 1920 indique clairement que la guerre constitue une rupture au niveau du nombre de prostituées qui semblent travailler à Bruxelles. En effet la multiplication par deux des malades vénériennes ne s'explique pas seulement par une hypothétique augmentation des maladies liées à la guerre, mais est aussi un indice que le nombre total de prostituées a augmenté.

12 La prostitution n'est pas limitée au centre-ville, mais doit être considérée dans les frontières du Grand Bruxelles, ensemble de communes qui envoient un délégué à la Conférence des bourgmestres ${ }^{11}$. En 1910, 394 prostituées sont inscrites sur le territoire de l'agglomération bruxelloise. La très grande majorité (87 \%) figurent sur le registre en tant que filles éparses c'est-à-dire ne travaillant pas dans des maisons closes. À côté de la commune de Bruxelles (40\%), ce sont surtout Schaerbeek et Uccle qui rassemblent un grand nombre de prostituées (ensemble $42 \%$ ). Malheureusement le rapport en question ne fournit pas de chiffres pour Saint-Josse-ten-Noode, commune qui comptait une forte communauté de prostituées ${ }^{12}$. Au XIX ${ }^{e}$ siècle plusieurs personnes regrettent le fait que les communes bruxelloises sont incapables de poursuivre une politique d'ensemble dans ce domaine; les prostituées semblent avoir tiré profit de la noncohérence des différents règlements : plusieurs d'entre elles habitent les faubourgs où il n'existe parfois pas de réglementation, mais travaillent à Bruxelles.

\section{La mise en place laborieuse d'un système de contrôle (août 1914 - février 1915)}

\section{a) Occupé et occupant : un couple qui se cherche}

13 Le 20 août 1914 les troupes allemandes pénètrent dans la capitale. Le gouvernement s'est retiré à Anvers. Le collège échevinal, avec à sa tête le bourgmestre Adolphe Max (1869-1939), est la plus haute autorité dans la ville; c'est lui qui négocie la reddition avec les responsables du $4^{\mathrm{e}}$ corps de l'armée allemande. Le 4 août, lorsque l'invasion de la Belgique commence, le ministre de l'Intérieur Paul Berryer envoie une circulaire à toutes les autorités communales du pays, dans laquelle il précise l'attitude de celles-ci lorsqu'elles se retrouveront sous l'autorité effective de l'occupant. «Elles demeureront donc en place et continueront à exercer leurs fonctions dans l'intérêt des habitants. 
Elles ne cesseraient ces fonctions que si l'envahisseur prétendait leur imposer des actes qui seraient directement contraires à leurs devoirs de fidélité au Roi et au pays. (...) Les autorités communales seront ainsi nécessairement en rapport quotidien avec les autorités étrangères. Celles-ci, disposant du pouvoir, seront tenues de prendre toutes les mesures qui dépendent d'elles pour assurer l'ordre et la vie publics. Les lois en vigueur dans le pays seront appliquées; elles ne pourront être modifiées ou abrogées qu'en cas d'empêchement absolu de les maintenir. (...) Le bourgmestre veillera avec un soin particulier au maintien de l'ordre et de la sécurité dans sa commune. S'il était nécessaire, il demanderait aux autorités étrangères de lui prêter main forte $»^{13}$.

Ces trois passages extraits d'un texte de deux pages indiquent clairement le cadre dans lequel les collèges communaux et leurs employés peuvent coopérer avec l'autorité ennemie. La circulaire ne laisse aucun doute quant à l'attitude à prendre une fois la commune occupée : il s'agit de maintenir la vie administrative au niveau local; une grève générale qui forcerait l'occupant à mettre en place un appareil administratif complet, est exclue. La coopération, aussi longtemps qu'elle ne met pas en cause la loyauté vis-à-vis du "gouvernement légal », est même souhaitée. Quelle aurait été la teneur du texte si le ministre de l'Intérieur avait su que l'occupation allait durer quatre ans? L'esprit de la circulaire s'applique plutôt à une courte guerre pendant laquelle il vaut mieux maintenir les structures administratives pour pouvoir reprendre le cours des affaires après une libération pas trop lointaine. On peut contrebalancer cette argumentation en relevant le fait que le gouvernement belge réfugié au Havre n'a jamais rectifié le tir par la suite. Lors de la première rencontre d'Adolphe Max avec des représentants du $4^{\mathrm{e}}$ corps d'armée, le capitaine Kriegsheim, chef de la délégation allemande, lui demande de rester en fonction. Max ne semble jamais avoir pensé à ne pas respecter la circulaire Berryer. Il rassure Kriegsheim en affirmant qu'il ne se contentera pas de rester en place mais qu'il va même tout faire pour garantir la sécurité des troupes allemandes qui traverseront et séjourneront à Bruxelles ${ }^{14}$.

Pour l'armée du Kaiser, cette attitude est tout bénéfice. Aucun plan concret par rapport à l'occupation d'un pays, d'une ville n'a été élaboré. La mise en place du Gouvernement général se caractérise d'ailleurs par un grand chaos. Les expériences faites pendant la guerre franco-allemande de 1870 serviront finalement d'exemple à suivre. Lorsque, par sa proclamation du 2 septembre 1914, le premier gouverneur général, von der Goltz ${ }^{15}$, annonce la création d'une administration allemande, il fait l'appel suivant : «Je vous invite à lui [gouverneur général] montrer de la confiance et à lui prêter votre concours. J'adresse cette invitation spécialement aux fonctionnaires d'État et des communes qui sont restés à leurs postes. Plus vous donnerez suite à cet appel, plus vous servirez votre patrie $»^{16}$. La volonté de collaborer existe donc des deux côtés.

$\mathrm{Au}$ niveau de la police dont dépend le contrôle de la prostitution, le principe de coopération est appliqué dès le début. Comme nous l'avons vu, le bourgmestre de Bruxelles lui confie dès le premier jour une tâche essentielle : assurer partiellement la sécurité du passage des troupes allemandes ${ }^{17}$. Or, Max dispose d'un corps de police fortement réduit, suite au départ de nombreux policiers en tant que soldats. Sur un effectif originel de 803 membres, 299 soit plus d'un tiers se sont engagés dans l'armée. La perte est surtout importante parmi les agents dont moins de la moitié reste à Bruxelles. Le service de la police des mœurs qui fait partie de la Division Centrale a probablement été peu (ou pas) touché. Depuis 1850, trois policiers dans chaque division se sont spécialisés partiellement dans cette tâche. En 1877, un service spécifique 
composé d'un commissaire adjoint, d'un agent spécial et de cinq agents inspecteurs est créé. Aucun agent inspecteur n'est parti; de même, peu de commissaires adjoints et d'agents spéciaux ont été enrôlés ${ }^{18}$. Le bureau des mœurs a donc probablement continué son service comme avant l'invasion. Il sera dirigé pendant toute la guerre par Joseph Broché qui est entré en service en 1886. Un questionnaire envoyé par les Allemands nous permet de découvrir comment le bureau des mœurs fonctionne pendant les premiers mois de l'occupation: au début de l'année 1915, son effectif est complet. Contrairement aux autres agents qui portent l'uniforme, le personnel porte une tenue civile pour «favoriser une surveillance plus discrète des femmes qui se débauchent tant sur la voie publique que dans les établissements publics ». Les sept agents sont secondés par deux médecins qui disposent d'une station médicale exclusivement utilisée pour la visite des prostituées. En cas de maladie, celles-ci sont envoyées à l'hôpital St-Pierre où il existe de nouveau un quartier spécial, séparé des autres services, pour les femmes. Elles y sont retenues jusqu'à leur guérison complète. Les visites sont obligatoires et ont lieu deux fois par semaine. Les frais occasionnés sont à la charge de la caisse communale. En cas de non-respect du règlement, le tribunal de police peut infliger une amende de 5 à 25 francs et un emprisonnement de 1 à 7 jours. En janvier 1915, 161 prostituées sont inscrites dont 114 éparses et 47 dans les maisons de tolérance ${ }^{19}$.

\section{b) La prostitution comme révélateur d'une difficile cohabitation entre occupé et occupant}

17 À partir du 20 août 1914, Bruxelles est envahie par des soldats et des fonctionnaires allemands. Outre les agents de l'administration allemande qui commence à se mettre en place, la capitale est aussi un lieu de transit vers le front grâce à son nœud ferroviaire. Il est impossible de déterminer le nombre exact de personnes qui y habitent ou y passent du fait de la guerre. Mais il n'est guère douteux que la demande de prostituées ait fortement augmenté. En août 1916, plus de 21000 soldats allemands sont stationnés dans le Brabant dont 9200 dans le Grand Bruxelles. Dans le gouvernement général, Bruxelles est de loin la ville avec la plus grande population prostitutionnelle. Le nombre de prostituées y est presque égal à celui des deuxième et troisième villes du pays (Anvers et Liège) mises ensembles ${ }^{20}$.

18 Six jours à peine après l'entrée des troupes allemandes, le sujet apparaît déjà à la tribune publique. Lors d'une séance du conseil communal, le conseiller socialiste Camille Huysmans révèle la " conduite scandaleuse de certaines femmes ». La réplique de Max est particulièrement intéressante: «le bourgmestre répond qu'il a pris des mesures pour empêcher des faits de ce genre et qu'il a fait incarcérer plusieurs de ces femmes. Il ajoute qu'en vue de restreindre la cause de surexcitation, il y aura lieu d'ordonner la fermeture des cabarets à 9 heures du soir et d'interdire la vente d'alcool. Il importe qu'aucun acte de provocation ne soit commis. Les représailles pourraient être terribles $\aleph^{21}$. Max fait ici probablement référence à deux rafles organisées par la police dans le centre. Les ${ }^{22}$ et ${ }^{23}$ août, la brigade judiciaire arrête au total 26 femmes belges et françaises pour prostitution clandestine. Ce n'est pas la police des mœurs, avec un effectif réduit, qui s'en est occupé, mais la brigade judiciaire qui dispose de plus de membres et qui a une certaine expérience pour organiser des actions de plus grande envergure. La raison principale de cette conduite énergique ne se trouve pas dans un raisonnement moral (trahir la patrie) ou médical (risque de propagation de maladies 
vénériennes). Son intérêt principal est le maintien de l'ordre. En effet, la 'conduite scandaleuse' semble avoir choqué la population. Il craint que celle-ci s'en prenne à ces femmes et ainsi, indirectement, aussi aux soldats qu'elles accompagnent. "Les représailles pourraient être terribles ».

Les mois suivants démontreront que les craintes du bourgmestre ne sont pas dénuées de tout fondement. De nombreux rapports de police témoignent des tensions qui naissent lorsque les soldats allemands s'amusent dans les cabarets et bars belges. La police communale doit intervenir régulièrement pour disperser les foules qui se rassemblent devant ces locaux. De plus, il semble que le nombre de soldats soit supérieur pendant les semaines d'octobre et novembre 1914 par rapport à celui des années suivantes. Face à ces militaires, les agents sont impuissants. Il y a d'ailleurs un vrai mouvement de grogne fin 1914 parmi ceux-ci parce que leur autorité est régulièrement mise à l'épreuve devant le public belge. Or, la police militaire allemande en est uniquement à ses débuts; ce n'est qu'à partir de 1915 qu'elle sera répartie avec des effectifs suffisants à travers Bruxelles. Elle ne peut donc pas encore remplir effectivement son rôle. En octobre 1914, la maison d'une veuve située rue des Alexiens, un des coins les plus visités par les amateurs d'amour vénal, est entourée par un public hostile «sous le prétexte qu'elle recevait des Allemands ». La foule est dispersée une première fois par la garde bourgeoise, police auxiliaire créée au début de l'occupation. Quelques minutes plus tard, la veuve est agressée physiquement; l'intervention d'une patrouille allemande la sauve. Deux heures plus tard, des individus essaient de s'introduire dans sa maison et c'est une nouvelle fois des militaires allemands qui la défendent. L'un d'entre eux sort même un revolver pour rétablir l'ordre. Le rapport de police ne nous donne malheureusement pas d'indication sur le nombre de perturbateurs ou leur origine. La veuve en question parle de "voyoux (sic) du quartier (...) qui veulent l'ennuyer en provoquant des rassemblements devant sa maison ». La rue des Alexiens est pendant les derniers mois de l'année 1914 le principal lieu d'amusement des soldats allemands. Quelques semaines avant l'incident précité, des soldats y chargent le public à la baïonnette pour 'libérer' un cabaret tenu par un sujet de Guillaume II. Celui-ci provoque l'ire des habitants du quartier qui sont cependant habitués à ces scènes de 'débauche', parce que l'individu accueille de nombreux soldats ennemis ${ }^{23}$. À ce stade de la recherche, il nous est impossible de déterminer si de tels événements sont fréquents ou non. Cet exemple indique cependant que la prostitution ouverte avec les Allemands provoque au début de l'occupation la vive réprobation du public. Le témoignage de la veuve, même s'il est évident qu'elle ne va pas avouer être une prostituée, peut aussi laisser croire que le reproche de 'recevoir' des Allemands peut être un prétexte pour régler d'autres différends antérieurs à la guerre.

Malgré les deux rafles organisées tout au début de l'occupation et qui pourraient présager un engagement massif et permanent de la police, la prostitution et sa surveillance continuent de poser des problèmes aussi bien à l'intérieur de la police que parmi la population bruxelloise. Avant la guerre, les membres des différentes divisions ont secondé la petite équipe de la Division Centrale qui s'occupait de la prostitution. Or les $3 \mathrm{e}$ et $4 \mathrm{e}$ divisions qui sont le plus concernées par la problématique, sont confrontées à un manque de personnel. Ainsi un commissaire-adjoint s'en plaint le 1er octobre 1914 à Lemonnier ${ }^{24}$ tout en l'assurant de la bonne volonté de son service. Il souligne la faiblesse numérique de son personnel qui ne permet pas d'organiser une surveillance maximale ${ }^{24}$. Des lettres arrivent régulièrement à la maison communale dans lesquelles 
des plaintes relatives à des prostituées liées avec des Allemands sont formulées. Ainsi les habitants de la rue aux Fleurs, située dans le quartier 'chaud' de la 3e division, se plaignent de ces femmes "qui se livrent à la prostitution et qui ne marchent exclusivement qu'avec des soldats allemands et qui font des orgies sans nombre $"{ }^{25}$. Plusieurs enquêtes sont effectuées suite à des dénonciations anonymes, phénomène qui semble être particulièrement développé en temps de crise. En période de guerre, la prostitution est encore plus fortement stigmatisée qu'en temps de paix. Elle est à l'opposé de l'image qu'un pays en guerre se fait de lui-même. En Belgique occupée, ce premier niveau de condamnation est doublé par le reproche de coucher avec l'ennemi. Dans un pays coupé de son armée, le devoir patriotique est jugé particulièrement important. Il permet de poursuivre la lutte contre l'ennemi sur le home front ${ }^{26}$. La prostituée apparaît dans ce contexte comme traître.

\section{c) D'un contrôle des lieux jugé insuffisant par l'occupant...}

Du côté allemand, la surveillance de la prostitution par les autochtones ne satisfait pas les responsables militaires et administratifs. Dès le mois de septembre, la police militaire intervient dans la surveillance du monde des prostituées. Celle-ci effectue des contrôles au moins depuis la fin septembre 1914. Lors de ces patrouilles, elle se limite cependant à contrôler les soldats allemands et ne s'occupe pas des prostituées, pour la plupart belges. Lorsque la Kommandantur constate que des soldats sont tombés malades, elle s'adresse à la police bruxelloise en lui demandant de faire un examen médical. C'est le capitaine Fischer de la section XI de la Kommandantur qui est, du côté allemand, responsable pour cette question. Je ne dispose pas de beaucoup d'informations sur l'organisation institutionnelle de la Kommandantur et de la place que Fischer y occupe. Les premiers mois de l'occupation, il semble être l'intermédiaire entre les autorités communales et l'administration allemande pour la question du maintien de l'ordre. Ses interventions ne se limitent pas à l'amour vénal, mais concernent aussi le colportage, la circulation... Il n'y a pas à l'intérieur de la Kommandantur de service spécifique, responsable pour les prostituées avant la création de la Sittenpolizei (police des mœurs).

En novembre et décembre 1914, des premières mesures concrètes sont prises. Les six maisons de tolérance qui existent sur le territoire de la ville de Bruxelles, sont placées progressivement sous le contrôle particulier de la Kommandantur et mises à la disposition exclusive des soldats allemands. Mais contrairement à ce qui se passera pendant la Deuxième Guerre mondiale, les autorités militaires ne créent pas leur propre réseau de bordels militaires (Wehrmachtsbordelle), en tout cas pas dans le Gouvernement général. La situation est peut-être différente dans les zones d'étapes ${ }^{27}$. Ils recourent plutôt à des structures existantes sur lesquelles ils ont un droit de regard. D'après le témoignage d'un des propriétaires, les clients habituels belges sont à ce moment presque complètement absents de ces maisons ${ }^{28}$. Celles-ci reçoivent l'autorisation de rester ouvertes jour et nuit. Située dans la 4e division, la rue StLaurent dans laquelle se trouvent quatre des six maisons, reçoit ainsi un statut d'extraterritorialité'. La police bruxelloise ne peut y intervenir que sur réquisition de la police militaire allemande ${ }^{29}$. Cette mesure est motivée par deux réalités. D'une part de nombreux incidents éclatent entre soldats allemands et les rares Belges fréquentant les mêmes maisons de tolérance. D'autre part, la santé des troupes commence à inquiéter les responsables militaires. Désormais les deux mondes sont clairement 
séparés. De plus, le monde prostitutionnel est souvent perçu comme un lieu idéal pour les espions. L'ambiance décontractée du cabaret, l'intimité qui y règne, l'alcool : tout incite à ne plus se tenir sur ses gardes ${ }^{30}$. Le processus décisionnel semble témoigner d'un certain respect de part et d'autre. Les responsables de la Kommandantur expriment, début novembre, le désir de voir se créer des maisons spécifiques pour leurs hommes. Mais ils laissent aux autorités belges, notamment au commissaire Crespin ${ }^{31}$ qui se réunit régulièrement avec des membres de la Kommandantur, l'exécution pratique des mesures.

Ces mesures partielles permettent certes de contrôler les lieux de débauche, mais présentent toujours deux inconvénients. Les femmes qui y travaillent ne sont pas enregistrées de manière systématique. De plus, la plupart des prostituées ne travaillent pas dans ces maisons de tolérance. C'est cette réalité qu'entre autres Parent-Duchâtelet avait dénoncée qui rend la surveillance particulièrement difficile. Or, les rapports de police d'octobre et de novembre montrent que la prostitution 'sauvage' connaît une véritable explosion. Les rues de Bruxelles sont 'envahies' par les filles publiques. Un contrôle des lieux ne peut pas tenir compte de ces mouvements; il doit être remplacé par un contrôle des corps mêmes.

\section{d) ... à un contrôle du corps par l'occupant (février 1915)}

Le 13 février 1915 le gouverneur général von Bissing crée par arrêté une police des mœurs pour l'agglomération bruxelloise ${ }^{32}$. À peine un mois plus tard, Gerstein, le chef de cette section, publie un nouveau règlement intitulé 'Mesures de police relatives à la sécurité de la santé, de l'ordre et de la décence publics'. Ce changement de cap ne constitue pas une véritable surprise. Les signes annonçant cette intervention se sont multipliés à partir de janvier 1915.

Mi-janvier, Gerstein, président de l'administration civile allemande pour la province de Brabant, adresse une lettre à Maurice Lemonnier, le bourgmestre faisant fonction. Il souligne le fait que seules cent «filles de joie » sont encore enregistrées à Bruxelles et que ce nombre ne peut pas correspondre à la réalité. S'il s'intéresse à cette problématique, c'est pour des raisons sanitaires: il constate que le nombre des maladies sexuelles a fortement augmenté. À la fin de sa lettre, il souhaite être renseigné sur quatre points : l'existence d'un règlement sur la prostitution, l'organisation de la police de mœurs, le nombre des prostituées surveillées et les propositions pour améliorer le système mis en place ${ }^{33}$.

Dans une lettre que von Kraewel, gouverneur militaire de Bruxelles, envoie fin janvier 1915 à Lemonnier, il propose les trois mesures suivantes : création d'un cadre législatif qui permet une étroite collaboration entre la ville de Bruxelles et les faubourgs; augmentation du personnel travaillant au bureau des mœurs; amélioration de l'infrastructure médicale sous la direction (ou avec la coopération ?) du médecin Frede, directeur du service de dermatologie et des maladies vénériennes à l'hôpital militaire, avenue de la Couronne. Avant la guerre, Frede travaillait comme médecin de la police des mœurs à Berlin. Si von Kraewel reconnaît que le but initial est lié aux intérêts médico-militaires allemands, il souligne le fait qu'une réorganisation sera aussi bénéfique pour la population locale. 
pourraient expliquer ce changement de politique. Les autorités communales refusentelles de créer une structure unitaire pour toute l'agglomération bruxelloise ou faut-il plutôt chercher la cause de cette modification du côté allemand ? Le nombre de soldats touchés par des maladies vénériennes a-t-il pris de telles proportions que les militaires cherchent à placer complètement ce secteur sous leur contrôle ? Dès février 1915, il y a en tout cas des voix qui s'élèvent à l'intérieur du Reich pour mettre en garde contre les dangers qui attendent les soldats en Belgique. La réglementation est jugée trop laxiste. Freiherr von Schoen exige une poigne d'acier vis-à-vis de la population belge et une discipline d'enfer [sic] parmi les troupes d'occupation pour éviter le pire. Il reproche à von der Goltz d'avoir été trop faible pour imposer les mesures qu'on aurait dû prendre dès le début. Il s'inquiète aussi des effets que le comportement de certains officiers à Bruxelles pourraient avoir sur l'image de l'Allemagne à l'étranger. Moritz von Bissing, qui a entre temps remplacé von der Goltz, peut rassurer von Schoen: les premières mesures viennent d'être prises. Un extrait de cette lettre est particulièrement intéressant. von Bissing y reconnaît explicitement que les officiers, après avoir vécu des moments difficiles, ont le droit de se 'défouler' : la prostitution y est présentée comme un remède à l'isolement et aux violences subies sur le front. Deux ans plus tard, l'argumentation reste identique, mais devient encore plus directe. von Bissing a entendu dire plusieurs fois « que des officiers du front se reposeraient vite des lourdes impressions psychiques et physiques en goûtant à des légers divertissements à Bruxelles». Des limitations de la prostitution auraient donc des conséquences plus «nocives qu'utiles ». L'instrumentalisation de la femme est poussée à son paroxysme. Le sort des prostituées qui doivent supporter ces excès, n'est pas pris en considération ${ }^{34}$.

28 La décision d'engager des employés allemands pour la nouvelle police est en tout cas déjà prise fin janvier 1915. Dans une lettre du 1er février 1915, von Sandt informe le secrétaire d'État de l'Intérieur que les polices de Berlin, Cologne et Leipzig sont prêtes à lui fournir les hommes dont il a besoin pour le nouveau service ${ }^{35}$. L'administration allemande en Belgique a cette fois-ci mieux préparé le terrain que lors de la création de la police politique à l'automne 1914. À ce moment les autorités berlinoises avaient tergiversé en affirmant qu'il n'y avait pas de personnel disponible. Cette fois, la demande de création d'un nouveau service n'est adressée aux autorités impériales qu'à partir du moment où il est clair que le problème des effectifs ne se posera pas.

La Sittenpolizei est une institution dépendant directement du chef de l'administration civile. À sa tête se trouve Gerstein. Avant la guerre, il était président de la police à Bochum. Une ordonnance de police affichée le 13 février proclame que « [1]es femmes se livrant par profession à la prostitution sont placées sous le contrôle de [cette] police ». Elle fait aussi référence à un nouveau règlement qui n'apparaîtra qu'un mois plus tard ${ }^{36}$. Pendant ce mois le nouveau service n'est d'ailleurs pas encore opérationnel : il est dépourvu d'un local et des réunions préparatoires semblent encore avoir eu lieu entre les autorités allemandes et belges. Gerstein propose aux seize communes concernées de former un comité composé de trois personnes «de préférence parlant allemand [afin de] m'entendre avec ceux-ci pour discuter les frais d'organisation et d'entretien ${ }^{37}$. Ce n'est que le 3 avril que Friedberg, qui est le viceprésident de l'administration civile du Brabant, peut informer Lemonnier que la Sittenpolizei est entrée en fonction. Dans les mois suivant son instauration dans la capitale, le même système est institué à travers toute la Belgique. Le fonctionnement à 
Bruxelles semble être considéré comme exemplaire. Tous les agents doivent d'abord y effectuer un stage avant de partir à Anvers, Liège ou Mons ${ }^{38}$.

Le nouveau règlement a une composante essentiellement médicale. Il s'applique « dans le district de police de l'agglomération bruxelloise, aux femmes s'adonnant par profession à la prostitution». Cette nouvelle mesure est instaurée "par raison d'hygiène » et la police exerce essentiellement un "contrôle sanitaire ». Les quatre premiers articles règlent la visite médicale. Celle-ci se déroule à l'hôpital de Saint-Gilles et ne peut pas être refusée. En deuxième lieu, le comportement des prostituées en public est réglementé: défense d'avoir une "tenue provocante", défense d'habiter près d'une école, d'une église... Finalement les prostituées sont munies d'un carnet de contrôle qui permet leur mise en fiches. Elles doivent toujours l'avoir sur elles pour pouvoir "l'exhiber à la réquisition des fonctionnaires de police». Les frais sont à supporter par les seize communes, proportionnellement à leur nombre d'habitants ${ }^{39}$. D'après le Berliner Tageblatt, le règlement s'inspire essentiellement de la réglementation en cours à Leipzig et Berlin. À la fin du XIX siècle, le néo-réglementarisme était né en opposition au mouvement abolitionniste. Ce mouvement prône un renforcement du contrôle de la prostitution, non plus tellement à cause de la crainte d'une contagion morale mais par peur d'une contagion biologique. Ce courant, surtout défendu par des médecins, avait influencé la législation prussienne au début du XX $\mathrm{XX}^{\mathrm{e}}$ siècle ${ }^{40}$. Le texte de mars 1915 s'inscrit dans ce cadre. La prostitution n'est pas nécessairement contrôlée parce qu'elle incite à la débauche; pour l'armée en tant qu'institution ce n'est pas un phénomène nouveau. La prostitution constitue presque un élément constitutif du milieu qui se crée lors de la présence d'un grand nombre de soldats. Par contre, la contamination et l'immobilisation des militaires qui s'en suit, sont particulièrement nocives pour le bon fonctionnement de l'institution: il faut donc limiter le risque au maximum par un contrôle médical effectif qui ne soit cependant pas trop étroit pour ne pas faire fuir les femmes et créer une trop importante prostitution clandestine. Les prostituées restent des marginales, mais à l'intérieur et non pas à l'extérieur du système. C'est dans le dernier cas qu'elles constituent un véritable 'danger'.

31 La création de la Sittenpolizei et la réforme du règlement provoquent une réaction tant des autorités communales que des agents bruxellois concernés. Les autorités allemandes se montrent d'ailleurs étonnées de cette résistance, surtout qu'à Anvers la mesure a été bien accueillie ${ }^{41}$. Les seize communes de l'agglomération bruxelloise formulent leurs réserves sur deux plans. Le premier est d'ordre juridique. À ce moment, au début mars, elles espèrent encore pouvoir modifier la nature du nouveau système. La compétence de réglementer la prostitution est attribuée par une loi belge aux collèges échevinaux; d'après ceux-ci, les Allemands n'auraient pas le droit d'agir sans leur accord. Ensuite, ce ne sont plus les tribunaux belges qui statuent en cas de contravention. L'ordonnance du 13 février énumère seulement les amendes qui semblent être imposées par l'autorité allemande; il n'y aurait aucune possibilité de recours ${ }^{42}$. Mais cette argumentation n'est pas valable pour Gerstein: "Après que la force légale est passée de fait aux mains de la puissance allemande dans les territoires occupés de la Belgique, cette Puissance a, suivant l'article 43 de la convention de la Haye sur la Guerre sur Terre, le droit et l'obligation de veiller au maintien de l'ordre public. Les arrêtés donnés par Mr. le Gouverneur Général, en qualité de porteur (représentant) de la force allemande, ont force de loi $»^{43}$. Cette ligne de défense allemande sera appliquée pendant toute la guerre dans différents domaines. Un mois plus tard, lorsqu'il est clair que les Allemands ne vont pas revenir sur leur décision, 
Lemonnier essaie de limiter les engagements financiers que le nouveau système entraîne pour les budgets communaux. Il demande à Gerstein de réduire considérablement les effectifs qui se composent d'une part des personnes engagées dans l'hôpital Molière et d'autre part des agents engagés par la police des mœurs allemande. Surtout ce dernier service lui semble démesuré ${ }^{44}$. Comme nous allons le voir, l'administration civile allemande ne va nullement tenir compte de ces remarques. Le bureau des mœurs bruxellois écrit déjà avant l'introduction de la Sittenpolizei que «[t]oute organisation est perfectible, mais [que] les mesures existantes paraissent suffisantes ». La montée en flèche du nombre des prostituées est due non pas à un 'dysfonctionnement' du bureau des mœurs mais «au manque de travail » de larges couches de la société belge ${ }^{45}$. Par la suite, les agents se plaignent surtout du fait que le nouveau règlement donne trop de libertés aux prostituées. Celles-ci sont autorisées à fréquenter les cabarets. De plus, elles peuvent habiter à plusieurs dans un appartement ce qui facilite le vol à l'entôlage, ces femmes « formant une véritable toile d'araignée où viennent se prendre les particuliers tentés par les charmes de ces drôlesses $»^{46}$. Finalement le caractère amoral du règlement semble choquer des membres du bureau des mœurs; dans un rapport on peut lire que de «temps immémorial, la police des mœurs a toujours cherché à détourner de la prostitution les filles et femmes, tant cartées que clandestines $»^{47}$.

En mars 1915, von Bissing confère avec des représentants de sociétés d'assurances allemandes qui lui font des propositions pour lutter contre les maladies vénériennes ${ }^{48}$. En octobre de cette même année, une conférence est organisée à Bruxelles où se réunissent une cinquantaine de hauts fonctionnaires et médecins venant d'Allemagne et des territoires occupés à l'ouest. Il s'agit de faire un bilan provisoire de la nouvelle réglementation. Bruxelles semble d'ailleurs être considérée comme un lieu particulièrement approprié pour discuter de la problématique. En mars 1917, 200 aumôniers du front de l'ouest se réunissent dans la capitale pour débattre des implications religieuses de la prostitution ${ }^{49}$. À la conférence du 8 octobre 1915 quatre sujets se dégagent. D'abord, les mesures réalisées à Bruxelles jusqu'à cette date sont considérées comme exemplaires pour les autres parties de la Belgique. Les représentants d'autres villes se plaignent d'ailleurs du manque de moyens par rapport à Bruxelles. Pour certains des participants, la capitale est même considérée comme un laboratoire d'expérimentation dont les résultats pourraient plus tard être aussi appliqués en Allemagne. Ceci rejoint un deuxième point. Une partie des orateurs prônent l'introduction de la déclaration obligatoire en cas de maladie par le médecin et/ou la personne malade. En Allemagne, cette mesure n'a pas été votée lors des réformes législatives au début $\mathrm{du} \mathrm{XX}^{\mathrm{e}}$ siècle. Elle est maintenant exigée notamment par les autorités militaires comme Sauberzweig, gouverneur militaire de Bruxelles. Pour les défenseurs de cette mesure, le nouveau caractère de la guerre permet cet empiètement. Dans une guerre totale, les pouvoirs de l'État deviennent illimités. Les autorités médicales pourront ainsi 'tester' cette réglementation pour voir si le nombre de malades vénériens diminue ${ }^{50}$. Une troisième remarque concerne les stéréotypes qui déterminent l'image que les autorités allemandes ont de la Belgique. Pour eux, le pays se caractérise par un laisser-aller particulièrement dangereux dans ce domaine. Les maladies vénériennes y seraient particulièrement répandues. Depuis l'affaire de la traite des blanches (1879-1881) qui a eu un retentissement international, Bruxelles apparaît comme un lieu de débauche, renforcé par la proximité de Paris. L'occupant aurait donc une 'mission civilisatrice' dans un pays qui se caractériserait par un certain 
retard dans sa politique médicale ${ }^{51}$. Le caractère 'missionnaire' de l'occupation témoigne d'un certain sentiment de supériorité ambiant parmi les fonctionnaires allemands. Finalement tous sont aussi d'accord pour souligner que le travail doit être fait en coopération avec les autorités du Gross-Brüssel, pour reprendre leurs termes.

Même si l'autorité occupante n'installe jamais une structure institutionnelle comparable à celle du Grand Bruxelles pendant la Deuxième Guerre mondiale, elle conçoit tous les problèmes sous un point de vue global et ne tient pas compte des différences qui existent entre les 16 communes de l'agglomération. La ville de Bruxelles acquiert très vite un rôle prépondérant grâce à son administration qui doit centraliser une grande partie de la communication entre les faubourgs et l'administration régionale allemande. Un agent-inspecteur belge a assisté à la conférence (en tant qu'observateur ?). La seule mesure concrète qu'il peut rapporter au bourgmestre est l'envoi d'un questionnaire destiné aux communes bruxelloises et qui reprend d'une manière plus détaillée les questions que Gerstein a adressées à Lemonnier ${ }^{52}$.

\section{Le milieu prostitutionnel : un monde mouvant}

\section{a) Esquisse sociologique ${ }^{53}$}

Comme je l'ai déjà souligné, la grande absente des travaux en Belgique sur la prostitution est la prostituée. On ne dispose donc que de peu de chiffres de comparaison. Les données que je présenterai sont elles-mêmes à utiliser avec beaucoup de prudence. Les dossiers des filles contrôlées n'ont malheureusement pas été conservés. À la base de notre banque de données se trouve une liste de 511 femmes, établie par les Allemands au milieu de 1915. Elle reprend les prostituées qui se trouvent à ce moment sous contrôle médical ${ }^{54}$. Au fil de nos recherches, des données sur 250 autres 'filles publiques' ont été ajoutées ${ }^{55}$. L'échantillonnage reste néanmoins biaisé; il reflète d'abord la situation en 1915, c'est-à-dire au début de l'instauration de la surveillance allemande. Cette banque de données ne permet pas non plus d'observer des évolutions au cours des quatre années. De plus, de nombreuses catégories y sont probablement peu représentées comme les prostituées clandestines (par définition), les demi-mondaines, les femmes galantes, les femmes de théâtre... J'estime cependant que les résultats restent intéressants en tant que premières données - avec leur part d'incertitude - sur un monde difficilement saisissable. Aux travaux suivants de les affiner et, le cas échéant, de les corriger.

Tableau 1 - Prostituées enregistrées ${ }^{56}$

\begin{tabular}{|l|l|l|l|l|}
\hline & avril 1915 & juillet 1915 & octobre 1915 & juillet 1917 \\
\hline Grand Bruxelles & 556 & 836 & 1160 & \\
\hline Grand Bruxelles et Louvain & & & & 3855 \\
\hline
\end{tabular}

Il est impossible d'évaluer le nombre exact de femmes qui se sont livrées à la prostitution pendant la Première Guerre mondiale. La prostitution se caractérisant en partie par son côté clandestin, les chiffres 'officiels' ne représentent qu'un aspect de la 
réalité. Néanmoins, la hausse est réelle : entre avril et octobre 1915, le nombre de filles publiques double. Deux ans plus tard, il a encore au moins une fois doublé, Louvain ne pouvant pas être considéré comme un haut lieu de la prostitution. La multiplication par quatre n'est pas uniquement due à un contrôle renforcé; elle témoigne aussi de la forte croissance du nombre des prostituées.

Graphique 1 - Répartition par date de naissance $(n=706)$

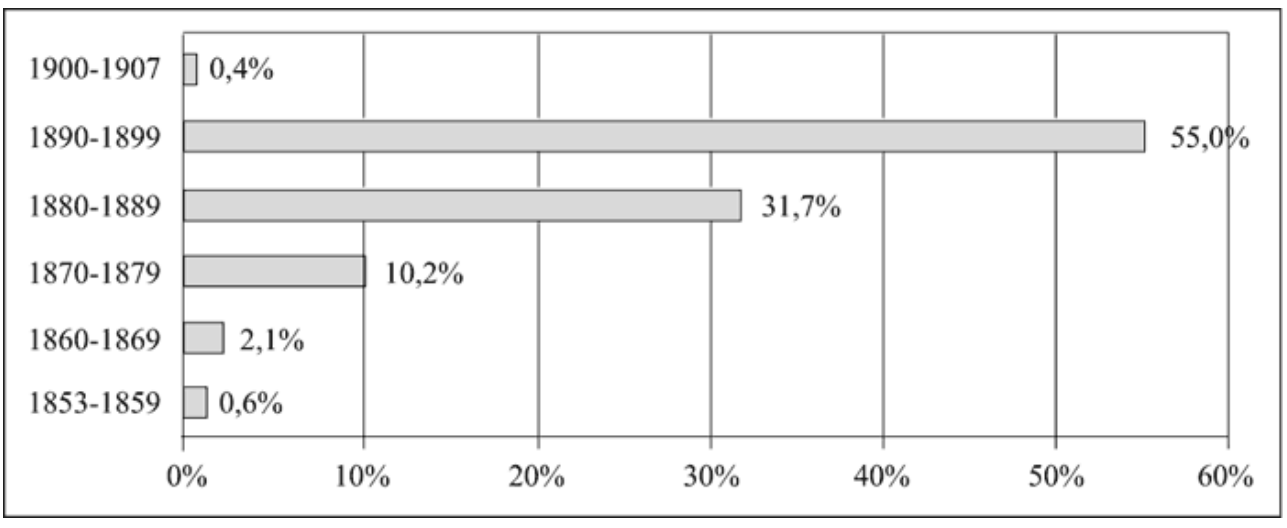

Les résultats sont à première vue contradictoires. Il y a d'un côté la relative jeunesse qui est un des faits marquants de la pyramide d'âge. La grande majorité des prostituées ont moins de 25 ans en 1916. La plus jeune est née en 1907 (10 ans en 1917 !). Le Naour relève aussi un rajeunissement conséquent des prostituées parisiennes au début de la guerre. Cette réalité s'explique entre autres par le bouleversement qu'entraîne le conflit. Les règles qui régissent la vie sociale et donc aussi la sexualité sont mises en question : quitter le cocon familial devient plus facile. Mais vu le chômage très répandu, les jeunes femmes qui décident de quitter leur environnement traditionnel, ont moins de possibilité de trouver un emploi susceptible de construire leur propre vie. D'un autre côté la moyenne d'âge (31 ans) est plus élevée que celle que Sophie De Schaepdrijver a relevée pour le dernier quart du XIX ${ }^{e}$ siècle $(25 \text { ans })^{57}$. Mais son échantillon contient seulement des femmes en maison de tolérance, et non pas des "éparses " qui en général sont moins jeunes. Une autre explication à cette moyenne est la présence particulièrement élevée de femmes mariées. Le taux de prostituées mariées (un huitième; $\mathrm{n}=589$ ) est deux fois plus élevé que celui déterminé par Corbin. Nous sommes probablement face à un caractère spécifique de la 'prostitution de guerre', la précarité poussant les femmes à vendre leur corps. Ceci constitue un des seuls indices dont on dispose pour voir d'où viennent les nouvelles prostituées. Dans le chapitre suivant, je vais revenir sur cette problématique. Ce dernier phénomène semble aussi avoir brouillé le comportement de la police des mœurs. Avant la guerre, la prostituée était facilement reconnaissable; pendant la guerre, il devient plus difficile de cibler les femmes potentiellement prostituées ce qui conduit parfois à l'arrestation par erreur de femmes «honorables et mariées ». Ceci fut déjà un des arguments les plus médiatisés contre la police des mœurs au XIX ${ }^{\mathrm{e}}$ siècle; et il n'est pas non plus absent du discours contre celle-ci pendant la guerre ${ }^{58}$. 
Graphique 2 - Lieu de naissance $(n=477)$

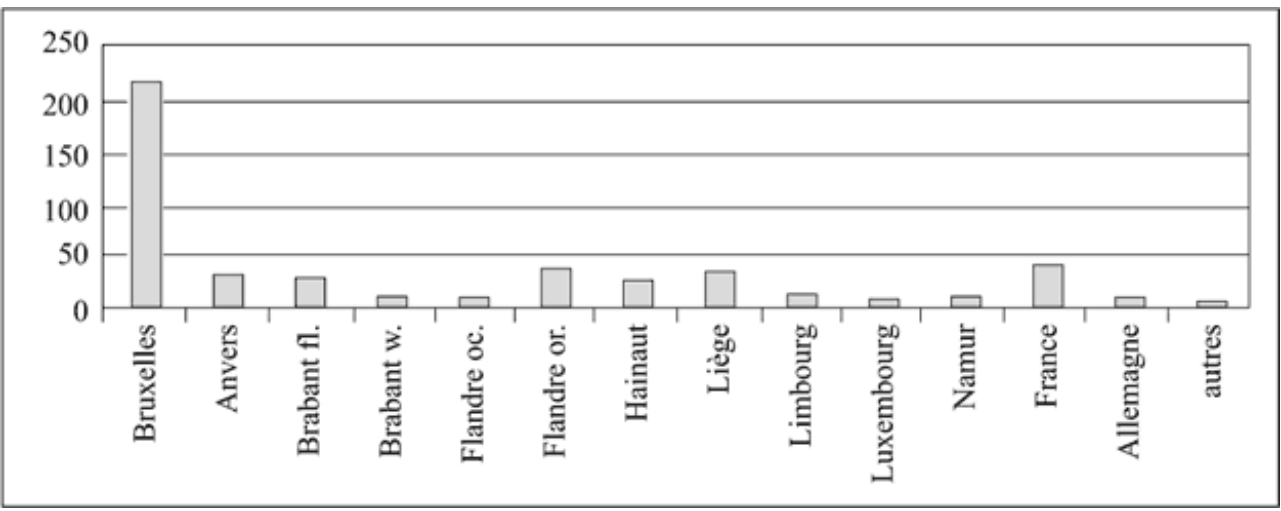

Pendant l'occupation, une très grande partie des prostituées viennent de l'agglomération bruxelloise. La France est la deuxième 'province'. Depuis la guerre franco-allemande en 1870, de nombreuses prostituées se sont installées à Bruxelles. De plus, la communauté française est de loin la plus importante parmi les communautés étrangères résidant dans la capitale. Avant la guerre, les prostituées françaises représentent $10 \%$ de la population prostitutionnelle. Pendant l'occupation, elles sont soumises à un contrôle spécifique à cause de leur appartenance à une nation en guerre avec l'Allemagne. Grâce aux listes établies mois par mois pendant toute la guerre, on voit que leur nombre double à partir de mi-février 1917, lorsque la Belgique, et Bruxelles en particulier, accueillent de nombreux réfugiés français dont la situation socio-économique se caractérise par une extrême précaritée $^{59}$. Le pourcentage d'Allemandes $(1,98 \%)$, plus faible qu'au XIX ${ }^{e}$ siècle, indique qu'il n'y a pas eu une 'importation' de prostituées d'outre-Rhin pour satisfaire les 'besoins' de leurs compatriotes. Ces résultats nous permettent de dégager une autre spécificité de la prostitution de guerre: un recrutement géographique plus réduit. Dans l'étude de Sophie De Schaepdrijver seulement $13 \%$ des prostituées venaient du Brabant, pendant la guerre ce pourcentage est supérieur à $50 \%$. Ceci n'est d'ailleurs pas étonnant, la croissance de l'agglomération bruxelloise au XIXe étant largement soutenue par une immigration des autres provinces belges et de l'étranger. Les résultats de Corbin, cette fois pour la France au XIX siècle, témoignent aussi de la grande mobilité des prostituées. À Paris, le chiffre des indigènes est de 27 \% entre 1880-1886. L'occupant a fortement réduit la mobilité entre régions pour mieux contrôler la population. Il est fort probable que l'augmentation du nombre des prostituées pendant la guerre soit essentiellement alimentée par des femmes issues du Grand Bruxelles.

La répartition dans l'agglomération bruxelloise, en se basant sur l'adresse des prostituées, donne l'implantation géographique du phénomène, même s'il faut garder en mémoire que le lieu d'habitation n'est pas nécessairement identique à celui du travail; de nombreuses prostituées gagnent leur vie à Bruxelles, mais habitent les faubourgs. La capitale reste de loin la commune la plus prisée $(46 \%, \mathrm{n}=376)$, suivie par Schaerbeek et Saint-Josse-ten-Noode. Ce qui est étonnant c'est qu'Uccle qui occupait une place importante avant la guerre, semble avoir perdu de l'importance. À l'intérieur de Bruxelles, trois des sept divisions de police (la 2e, la 3e et la 4e) rassemblent 95 \% (n = 289) des prostituées. Au centre de Bruxelles, la 2e division se caractérise par un relatif éparpillement. Trois rues retiennent l'attention: la rue de la Chaufferette, celle de la Gouttière et celle des Alexiens. Au nord du Pentagone, dans la 3e division, la 
prostitution se concentre entre l'actuel boulevard Émile Jacqmain et la rue de Laeken avec la rue St-Roch comme centre. La 4e division où habite presque la moitié des femmes, présente de nouveau une répartition plus dispersée. C'est dans cette division qu'est située la voie publique la plus 'mal famée', la rue St-Laurent. Un quart des prostituées y habitent. Les rues Vander Elst et St-Pierre sont d'autres endroits où on peut observer une concentration de filles publiques. Il semble qu'il s'agit des mêmes quartiers qui, déjà avant la guerre, attiraient le milieu prostitutionnel.

\section{b) La pratique prostitutionnelle}

La 'succursale de Paris', comme Bruxelles est appelée par les militaires allemands, est la plus grande ville occupée sur le front ouest. À côté de l'appareil administratif du Gouvernement général, composé essentiellement d'hommes, ce sont surtout les soldats venant du front qui remplissent le soir les rues de la ville. Laissons un journaliste hongrois raconter leur arrivée à la Gare du Nord : « Most of the men have just arrived from the Front, and on their way they're staying for a few hours in this fine city of Brussels. They stroll along the streets and do all the sightseeing they can, so that when they arrive home they may have something else to speak of than the horrors of the trenches. And, of course, one great feature of their stay here is the buying of keepsakes for 'mother and the kiddies'. Money is fairly plentiful among them, as for many weeks passed in the firing-line they have been unable to spend a penny $»^{60}$. Mais ils ne cherchent pas uniquement des articles de souvenir et des histoires à raconter à la maison.

41 Dès leur arrivée en ville, les soldats sont en effet entourés par des proxénètes qui essaient de les convaincre des avantages de 'leurs' filles. Les autorités allemandes se plaignent régulièrement de ces 'souteneurs' à la police belge. En septembre 1916, Gerstein propose même de régler le racolage. S'il en reconnaît la nécessité, il propose de confier à quelques personnes particulièrement dignes de confiance, l'autorisation de conduire les soldats dans les quartiers. Ceux-ci porteraient un brassard spécifique, un signe qui garantirait la 'qualité' de leur service. Ce projet ne semble cependant pas avoir été mis à exécution ${ }^{61}$. Ces hommes disposent le plus souvent de petites affiches publicitaires et parfois même de photos pour vanter la 'marchandise'. Sur une de ces photos $^{62}$, l'aspect marchand apparait clairement. On y voit une femme nue, de face, qui masturbe un homme également entièrement nu. Il s'agit d'une photo posée : le décor est celui d'un studio de photographie; la femme dont les cheveux sont soigneusement coiffés, est photographiée de telle sorte que son corps soit bien visible. La photo frappe par son côté 'froid' : il ne s'agit pas d'une mise en scène particulièrement érotique; le 'client' potentiel doit être capable de se faire une image des qualités physiques de la prostituée. D'autres prostituées n'emploient pas d'intermédiaire, mais se promènent sur la place Rogier située devant la gare du Nord ou sur le boulevard et la rue Neuve, non loin de ce nœud ferroviaire.

Comment reconnaît-on une prostituée ? Le soldat, arrivé dans les quartiers 'chauds', peut facilement la repérer par son habitus particulier qui la distingue clairement du reste de la société. Les rapports de police sont particulièrement intéressants à ce niveau. Les agents se basent essentiellement sur deux critères pour savoir s'ils se trouvent en face de prostituées: leur apparence physique et leur comportement. Portant «des toilettes voyantes », elles se promènent «très décolletées ", « habillées 
d'un vêtement très léger ", « les cheveux sur le dos ». Mais ce ne sont pas seulement ces indices purement 'physiques' qui les distinguent. «Leur attitude " montre " clairement qu'il s'agit là de filles qui se livrent clandestinement à la prostitution ». Quelle est cette attitude? Elles "fixaient des militaires allemands", "fumaient la cigarette», se tiennent assises sur le seuil de leur maison "les jambes étendues sur le trottoir", "frappaient sur les carreaux de vitre " pour attirer l'attention des passants ${ }^{63}$. Le XIX ${ }^{\mathrm{e}}$ siècle se caractérise par une forte 'disciplinisation' du corps (surtout féminin) dans toutes les couches sociales. Les gestes permis et interdits sont de plus en plus codifiés, même par écrit. Les livres de savoir-vivre se multiplient. Le processus de la "sexualisation des standards moraux" (Lorenz) est aboutie. Le corps disparaît définitivement sous une épaisse couche de vêtements. Cette 'tabouisation' est particulièrement forte dans la vie privée ${ }^{64}$. Or, comme le montrent les quelques extraits cités, la prostituée brise justement ces règles. Elle se marginalise elle-même - pour devenir reconnaissable - en exécutant des gestes qui ne correspondent pas aux codes établis. Même le fait de fumer en public est à ce moment encore tellement sexué qu'il est souvent un premier indice avancé par les agents de police pour justifier leur intervention.

Les archives sont peu parlantes en ce qui concerne la prostitution qui se déroule dans les maisons closes; la vie quotidienne des filles de rues est mieux documentée. Même si le soir et la nuit sont les périodes où la demande est la plus forte, l'activité prostitutionnelle ne se limite pas à ces heures. Une fois un accord conclu, la prostituée emmène son client dans son petit appartement ou dans une maison de passe où le client loue alors une chambre. Parfois l'acte se déroule dans la rue même. Les prix varient entre 2 et 6 marks, le prix d'une chambre entre 2 et 3 marks. Les officiers allemands par contre paient entre 20 et 50 marks. À Bruxelles, il n'y a pas de bordels réservés exclusivement aux officiers. Mais d'un autre côté, il est évident que vus les prix demandés dans certains bars, une certaine séparation sociale s'installe. Les prostituées ne semblent pas se spécialiser toutes selon la hiérarchie sociale de leurs clients. Elles adaptent simplement leur prix. Une prostituée fait parfois jusqu'à six passes par nuit. Dans les rares témoignages qui nous sont parvenus (procès-verbaux lors de vols à l'entôlage) l'acte se déroule toujours de la même façon: la femme et l'homme se déshabillent séparément, l'acte sexuel se déroule alors très vite: après 15-20 minutes le client quitte la chambre.

Pour échapper au contrôle policier et à la stricte réglementation des débits de boissons, le phénomène des clubs privés connaît une véritable explosion. Pour illustrer leur fonctionnement, j'ai choisi un cas sur lequel la documentation est un peu plus riche: situé au centre de Bruxelles, ce cercle présente une façade tout à fait honorable. Les statuts annoncent que le but «est de grouper tous ceux qui veulent s'occuper d'arts, littérature, musique, peinture, etc.»; le tout est chapeauté par un conseil d'administration. En fait, il s'agit d'un lieu de rendez-vous pour « un certain nombre de femmes de mœurs faciles, des joueurs et des gens qui cherchent à s'amuser ». Il ouvre ses portes lorsque les théâtres et autres établissements publics doivent fermer. Une liste de gens le fréquentant nous montre qu'il est aussi un lieu de sociabilité des élites urbaines. Tenu par une personne qui dirige la Spitzenzentrale ${ }^{65}$, on y rencontre aussi bien des négociants, des avocats, des entrepreneurs, des commerçants, un substitut du procureur du roi ou un vice-consul de Hollande que des Allemands. Vu la grande influence du propriétaire à l'intérieur de l'administration occupante, même la police allemande semble impuissante ${ }^{66}$. Rares sont les cas où nous disposons d'une 
documentation aussi précise. Néanmoins, ce monde interlope est un lieu de rencontre potentiel aussi bien entre l'occupant et l'occupé qu'entre les anciennes et les nouvelles élites belges (Zweepbarone ${ }^{67}$ ).

Comment se passe l'entrée dans la prostitution? Dans le chapitre précédent, j'ai relevé deux faits à savoir la jeunesse des prostituées bruxelloises et la précarité, qui sont des ébauches de réponse. La débauche de mineures semble être assez répandue. Des 'maisons' spécialisées et connues des militaires se créent rapidement. La 'première fois', les jeunes filles sont parfois accompagnées par une de leurs copines habituée depuis plus longtemps à ce genre de métier. Ce premier acte sexuel se passe souvent d'une manière douloureuse; les filles, âgées en moyenne de 15 ans, ne peuvent pas (ou ne veulent pas) nommer ce qui leur arrive. Le non-dit est encore renforcé par le mutisme de la société auquel elles se heurtent lorsqu'elles veulent témoigner ce qu'elles ont vécu. La déposition d'une fille âgée de 14 ans semble être symptomatique : « il m'a prise dans ses bras et mise sur le bord du lit; malgré que je me débattais et le priais de ne rien faire, il a retiré son affaire de son pantalon m'a ouvert de forces mes jambes et a introduit quelque chose dans mes parties sexuelles, $\underline{\mathrm{il} \text { a fait de sales choses }}$ et lui disant qu'il me faisait mal, il s'est retiré. (...) J'ai parlé de cette affaire à mon institutrice Madame Hermans, qui m'a conseillé d'oublier le passé, de bien me conduire à l'avenir ${ }^{68}$. Particulièrement les jeunes prostituées sont parfois tenues en 'esclavage' : "Nous sommes allées prendre un café que Triboulet a payé. Au cours d'une conversation, Triboulet m'a invitée à aller habiter chez elle (...) pour aller avec des hommes. J'ai quitté ma mère et je suis allée chez Triboulet où j'occupais une petite chambre (...) elle procurait des hommes à qui je me livrais moyennant 3 à 6 marks que je remettais à Triboulet. Elle payait ma chambre et me donnait la nourriture ${ }^{69}$. Cette fille de 14 ans a peu de chances d'échapper à cet univers: sans argent propre, sans autre contact social que la femme Triboulet, elle devient complètement dépendante de celle-ci.

En période de guerre, la misère, la faim, mais aussi le relâchement de l'autorité familiale poussent beaucoup de jeunes à sortir de leur environnement traditionnel. L'absence éventuelle du père, l'incapacité des parents à subvenir encore aux besoins de leurs enfants poussent ceux-ci à chercher d'autres repères ${ }^{70}$. Les jeunes femmes qui quittent leur cadre familial à cause de disputes, arrivent en ville sans savoir où dormir ni quoi faire. Une fille de 14 ans qui s'enfuit de chez elle, en témoigne : « ne sachant pas où aller, j'ai mendié pendant trois nuits. Le soir j'allais dormir dans une charrette (...) Il y a huit jours, j'ai rencontré à la Porte de Louise, un vieil homme qui m'a demandé de l'accompagner. Il m'a promis de me rendre heureuse (...) Il m'a donné à manger et je suis restée avec lui pour dormir avec lui dans le même lit. Pendant la nuit il voulait m'utiliser (sic !) $\gg^{71}$. Souvent c'est aussi l'amoureux de la fille (en fait un souteneur) qui lui fait abandonner le toit familial et la force ensuite à se prostituer. Dans ce cas de figure, les parents s'adressent parfois à la police en portant plainte. Le fait d'avoir un 'bouc émissaire' semble faciliter l'appel à cette institution pour régler un problème qu'on préfère souvent résoudre à l'intérieur de la famille ${ }^{72}$. Le maquereau n'apparaît dans les rapports de police que lors de faits de violence. Ces quelques procès-verbaux ne permettent ni une analyse précise de son origine sociale, ni une description de ses rapports avec la prostituée. À la lecture des archives, il ne faut cependant pas tomber dans une attitude trop réductrice. L'acte sexuel et la prostitution ne sont pas toujours perçus comme traumatisants. Une jeune fille de quinze ans affirme que la «maison Debeu est très connue parmi les jeunes filles de mon âge (...) pour pouvoir y obtenir des 
chambres à l'effet de $\underline{s}^{\prime} y$ amuser $\aleph^{73}$. Certes, ces quelques exemples ne sont pas forcément représentatifs de l'entrée dans le monde prostitutionnel. Ils permettent cependant d'aborder un sujet, les pratiques sexuelles, sans « se couvrir de la tunique de Noé (...) L'histoire sexologique (...) doit être une histoire du désir, du plaisir et de la misère sexuelle ${ }^{74}$. Le discours aseptisé de l'historien enlève souvent ces composantes essentielles de la prostitution.

Une caricature de Stadler publiée dans le livre de Hirschfeld montre une autre réalité de la prostitution en temps de guerre ${ }^{75}$. En échange d'un pain, une femme se laisse tapoter les seins par un soldat d'apparence plutôt désagréable mais bien nourri. C'est la précarité qui est régulièrement évoquée dans la littérature contemporaine comme cause première de la prostitution. Le chômage, la cherté et la rareté des produits alimentaires sont des réalités. Entre juillet 1914 et septembre 1918, le prix d'un œuf a été multiplié par quatorze, celui d'un kilo de farine par trente, celui d'une chemise par douze. Aussi bien les observateurs étrangers que les Belges partagent une pensée que le docteur Bayet, néoréglementariste actif avant la guerre, résume ainsi : "Cela ne me paraît pas étonnant, pour moi qui sais que la misère crée la syphilis. La misère n'est jamais assez générale pour qu'il n'y en ait pas qui aient de l'argent alors que d'autres ont faim. Dans ces conditions, la femme qui a faim se vend. Cela se voit en temps de paix, a fortiori en temps de guerre et c'est ce genre de femmes qui, à beaucoup près, est le plus dangereux $»^{76}$. Les archives semblent confirmer cette opinion, même si les exemples concrets sont plutôt rares. Ainsi une servante de Nivelles qui est licenciée après avoir travaillé pendant trois semaines, se retrouve - au sens littéral du terme - à la rue. Ne dénichant pas un autre emploi, elle est réduite à se prostituer ${ }^{77}$.

Les prostituées sont aussi vecteurs de pratiques qui semblent avoir été peu fréquentes en Belgique avant-guerre : la consommation de cocaïne. Contrairement à la police belge qui pendant longtemps refuse de consacrer du personnel dans la lutte contre cette drogue, les autorités allemandes s'en préoccupent très vite. Elles en craignent les effets sur l'élite de leur armée. $\mathrm{Vu}$ son prix, la 'gueuse blanche' semble particulièrement répandue parmi leurs officiers et leurs hauts fonctionnaires. La forte communauté d'artistes allemands d'avant-garde n'y est probablement pas étrangère. Le poète allemand Gottfried Benn, travaillant à Bruxelles comme médecin militaire, semble en tout cas y avoir consacré une partie de son temps et de son argent afin de ne pas trop s'ennuyer à l'arrière du front. Ce sont les prostituées ou leur souteneur qui en assurent la distribution. Outre son aspect prétendument aphrodisiaque (hypersensibilité des sens), la poudre blanche doit également son succès à un effet de mode. Entre 1914 et 1918, un vrai mythe se construit autour de la 'coco'. Pour les distributeurs, les bénéfices peuvent être considérables (prix d'achat d'un gramme : 1,75 frs; prix de vente : 2,5 frs) ${ }^{78}$.

49 Les plaintes déposées lors des vols à l'entôlage nous révèlent quelques indications sur la clientèle des prostituées bruxelloises. Certes, le nombre de clients identifiés est faible et la représentativité de l'échantillonnage est donc réduite. Néanmoins deux hypothèses peuvent être formulées. La clientèle de loin la plus importante est constituée de soldats allemands. Le réservoir géographique de clients potentiels ne se limite pas aux militaires stationnés à Bruxelles; les soldats de passage ou en congé venant du front représentent aussi un nombre non négligeable (ainsi il y avait des personnes venant de Coblence, Valenciennes, Moorslede, Namur, Savigny). Il semble bien que les Belges représentent une infime minorité. Plusieurs explications sont possibles: beaucoup 
d'hommes ont quitté Bruxelles; il y en a donc d'abord moins et ceux qui restent, trouvent peut-être plus facilement une compagne à l'extérieur du monde prostitutionnel. Les Belges sont peut-être aussi plus réticents à porter plainte.

\section{Le contrôle des prostituées : instructions et réalité}

\section{a) Une coopération difficile entre polices allemande et belge}

50 J'ai décrit dans un chapitre précédent la création de la Sittenpolizei et la mise en place d'un nouveau règlement. Comment ce nouveau système théorique est-il mis en place dans la pratique? Comment les différents acteurs réagissent-ils face à ces changements? Quels sont les résultats de ce contrôle? Telles sont les questions auxquelles je tâcherai de trouver une réponse.

51 La police des mœurs allemande restera pendant toute la guerre un service aux effectifs assez réduits. En 1915, celui-ci compte onze agents de police. À la fin de la guerre il se retrouve même réduit de deux unités; l'armée allemande ressent un besoin grandissant d'hommes; il en résulte un manque chronique de personnel pour ses administrations dans tous les territoires occupés. Ladite police dépend de l'administration civile régionale; le président de cette institution, Gerstein, est donc son chef immédiat. Même s'il dispose dans son administration d'un autre collaborateur qui s'occupe exclusivement de ce service, un assesseur du Gouvernement du nom de Friedberg, Gerstein semble avoir à cœur cette problématique. En tout cas, il intervient souvent comme en témoigne la correspondance échangée avec l'administration communale. Dans le quotidien, la Sittenpolizei est dirigée par deux commissaires. Le premier s'appelle Gebhardt; avant la guerre ce docteur en droit était fonctionnaire de la ville de Leipzig. En Allemagne, il n'est pas rare que des commissaires en chef aient une formation universitaire plus poussée. Le deuxième, répondant au nom de Galzow, travaillait à la police berlinoise avant de venir à Bruxelles ${ }^{79}$. Le règlement imposé par Gerstein à Bruxelles est justement inspiré de ceux de Leipzig et Berlin. Bruxelles est divisée en huit divisions. Comme je l'ai déjà souligné antérieurement, les Allemands ne tiennent pas compte du paysage administratif d'avant-guerre. Pour eux, le découpage du Grand Bruxelles en 16 communes ne semble correspondre à aucune logique; ils le considèrent comme contraire à l'organisation rationnelle d'un système de surveillance. L'unification des règlements et la centralisation de l'appareil policier est dès lors une des priorités de l'administration civile allemande. von Sandt estime d'ailleurs que cette mesure peut compter sur le support des autorités communales, vu que cette centralisation serait un souhait caressé depuis longtemps ${ }^{80}$. À la tête de chaque division nouvellement créée se trouve un policier allemand. Celui-ci est la plupart du temps un agent de la police criminelle. Des petites équipes mixtes composées du responsable allemand de la division et deux policiers belges surveillent leur territoire. Le déplacement en Belgique occupée présente deux grands avantages pour ces employés : ils échappent d'abord aux dangers du front; ensuite un emploi dans cette administration est à ce moment très demandé vu les possibilités d'ascension sociale. Ainsi, pour assurer leur préséance sur les policiers belges qu'ils rencontrent, ils bénéficient d'un relèvement de leur grade pendant la durée de la guerre ${ }^{81}$.

Cet effectif ne permet pas d'assurer un contrôle réel. La police des mœurs allemande a besoin de la coopération belge. Or, sur ce plan, les sources ne sont pas très parlantes. 
Prenons d'abord les quelques documents allemands qui abordent cette problématique. Gerstein utilise les mots suivants pour décrire la façon de fonctionner : «Le service est établi de telle manière que les employés allemands portent la responsabilité pour l'exécution conforme des affaires; des employés belges leur ont été transférés pour l'exécutif. Ils coopèrent avec ces derniers au bureau et dans la division et en assurent la surveillance $»^{82}$. Les explications de Schaible, le chef de l'administration pour la Flandre, trois ans plus tard ne sont pas plus explicites : «Le service [de la police des mœurs] à Bruxelles est en effet assuré non seulement par des employés allemands, mais aussi par des agents de police belges; mais pour la surveillance des rues (le service le plus important de la police des mœurs) il faut toujours les faire accompagner par des employés allemands pour s'assurer que les intérêts allemands seront sauvegardés ${ }^{83}$. Il s'agit en fait d'éviter que la police bruxelloise ne se montre trop expéditive vis-à-vis des officiers allemands.

Les archives belges sont un peu plus explicites. Dans une note rédigée en 1928, le chef du bureau des mœurs affirme que chaque commune de l'agglomération déléguait des policiers chargés de la répression de la prostitution à la Sittenpolizei. Le bureau des mœurs de la capitale y envoie cinq agents-inspecteurs (pour rappel le service se compose en tout de 7 agents). Pendant leur service les policiers belges sont en fait entièrement sous commandement allemand comme en témoigne le procès-verbal suivant: " Le 11 de ce mois, vers 9 heures du soir faisant notre service de surveillance accompagné du policier allemand Donachowski Théodore et de l'agent brigadier (...) notre chef Donachowski a dit d'aller voir au coin de la rue Berlaimont et de la rue des Comédiens où nous avons constaté le va-et-vient de jeunes filles (...) Sur l'ordre de notre chef de groupe précité, nous y sommes entrés ». Le policier allemand continue de mener l'action, dirige les policiers belges et interroge finalement les filles arrêtées ${ }^{84}$.

En fait, le contrôle de la prostitution n'est pas exercé par la Sittenpolizei seule comme on aura pu le croire en se limitant aux textes fondateurs de l'institution. Elle n'est pas non plus un simple organe d'inspection des différents services de mœurs existant sur le territoire du Grand Bruxelles. Une (petite) partie du personnel de police belge est mise à son service. L'administration communale n'abandonne pas uniquement une de ses compétences ( $c f$. la loi communale du 30 mars 1836), mais met ses employés à la disposition de l'autorité occupante. Ils continuent d'être payés directement par la ville de Bruxelles, mais ne semblent plus dépendre hiérarchiquement du commissaire en chef de la Division Centrale. À part les 5 agents délégués par la ville de Bruxelles, au moins 12 policiers venant des faubourgs sont envoyés tous les jours dans la rue des Long Chariots où la police allemande a son siège ${ }^{85}$. Et ils s'intègrent facilement dans cette institution allemande; leur travail n'a pas fondamentalement changé. Dans un rapport écrit quelques mois après la mise en place de la police des mœurs allemande, Sandt les trouve appliqués et qualifie leur travail d'impeccable ${ }^{86}$. Les dirigeants de la Sittenpolizei semblent ne plus tenir compte des propositions et plaintes transmises par le bureau belge des mœurs. Lors d'une formation que les agents délégués suivent sous la direction de Gebhardt, ils reçoivent comme instruction «qu'aucun fait ayant trait à la débauche ne pouvait être communiqué à la police belge et que toutes les réclamations portées à sa connaissance concernant les filles publiques seraient instruites par lui et recevraient la suite qu'elles comporteraient ${ }^{87}$. Néanmoins les agents bruxellois semblent en partie transgresser cet ordre parce que des rapports adressés à Crespin se retrouvent dans les archives de la police bruxelloise. Ces agents ne sont plus limités dans leur travail par l'appartenance à une des 16 communes; ils ont 
le droit de poser des actes officiels dans toutes les communes du Grand Bruxelles et de demander la coopération des employés de toutes les communes ${ }^{88}$.

Mais la collaboration entre polices belge et allemande ne se limite pas à l'envoi de ces cinq agents. En mars 1915, les Belges dressent, sur ordre allemand, une liste des "établissements suspects au point de vue de la prostitution clandestine". L'administration réalise un important travail de vérification d'identités grâce aux registres de population. Elle surveille l'application des mesures de fermeture édictées par les Allemands à l'égard des maisons de débauche et effectue des surveillances pour le compte du service social allemand (Fürsorgedienst). La Sittenpolizei s'adresse à Lemonnier lorsqu'elle désire organiser des rafles pour avoir des agents supplémentaires à sa disposition. Pour libérer les boulevards des prostituées, les postes de police militaire qui sont disséminés à travers Bruxelles, interpellent directement les commissaires des sept divisions. Dans ce cadre, il y a aussi une collaboration renforcée entre les polices des différentes communes. Normalement, la police d'une commune n'a pas le droit d'intervenir sur le territoire d'une autre commune. Or pendant la guerre, une coopération, toujours sur ordre de l'autorité occupante, se met en place. Ainsi les corps de Bruxelles et Saint-Josse-ten-Noode coordonnent leurs actions autour de la gare du Nord. Les 2e, 3e et 4e divisions continuent aussi d'envoyer des rapports à la Sittenpolizei. Elles y relèvent des contraventions et demandent à la police des mœurs allemande d'intervenir. Cette forme de surveillance est assez soutenue : ainsi entre le 5 et le 26 octobre 1916, 18 rapports sont envoyés à la rue des Longs Chariots; contrairement à ce qu'on pourrait penser, la police allemande ne l'apprécie pas. En décembre 1916, celle-ci déclare qu'elle ne tiendra plus compte à partir de cette date de ces rapports et qu'elle est seule responsable pour le service de la prostitution ${ }^{89}$.

$\mathrm{Au}$ début de la mise en application du règlement, le contrôle de la prostituée se caractérise par un certain chaos. L'inscription sur le registre des mœurs communal reste-t-elle obligatoire ? Début mai 1915, le collège échevinal de Saint-Josse-ten-Noode constate que "les administrations communales ne sont pas entièrement fixées sur l'étendue que l'autorité occupante entend donner à ses ordonnances sur les mœurs ". L'inscription des filles publiques dans les bureaux de la population est momentanément interrompue. Avant 1914, il leur était défendu de s'y rendre et leur inscription était effectuée par le bureau des mœurs. Lemonnier prend, en mai 1915, la décision de ne plus faire dresser de procès-verbaux en cas d'infractions. Les agents doivent par contre continuer de rédiger des rapports en double expédition, un pour l'autorité communale et un pour la Division Centrale ${ }^{90}$.

Le texte de mars 1915 sera seulement légèrement modifié pendant les trois années suivantes. Comme l'a proposé le bureau de mœurs, il sera défendu aux prostituées d'habiter à plusieurs dans la même maison. En septembre 1916, Hurt, le gouverneur militaire, fait exécuter une mesure qui rompt avec l'approche purement médicale que l'administration allemande a poursuivie jusqu'à ce moment. Il interdit en effet les grands boulevards du centre à la prostitution. Cette mesure ne peut avoir d'autre but que de rendre les prostituées moins visibles ${ }^{91}$. Les causes plus profondes de cette mesure 'morale' restent dans l'obscurité. Les femmes qui contreviennent audit règlement dépendent d'une juridiction allemande qui n'est pas spécifiée. Je n'ai trouvé que peu d'informations sur le fonctionnement de celle-ci et sur le nombre de femmes condamnées. Jusqu'en 1916 des traces de ces jugements sous forme de notices envoyées à Crespin, se retrouvent dans les archives belges. Grâce à un arrêté de condamnation 
conservé, nous savons que c'est Gerstein en tant que chef de la police des mœurs allemande qui prononce la peine. Un recours peut être introduit auprès du chef de l'administration allemande près du Gouverneur Général ${ }^{92}$. En cas de peine de prison, les prostituées, comme toutes les autres femmes condamnées, sont incarcérées à la prison de Forest.

Mais la police bruxelloise semble essayer de maintenir son système de surveillance propre. En tout cas, plusieurs plaintes de Gerstein témoignent de cette intention. Les divisions les plus concernées par la prostitution continuent d'envoyer des rapports à la Division Centrale. Elles peuvent même, indirectement, punir les femmes qui se livrent à la prostitution clandestine. Si celles-ci sont secourues d'une manière ou d'une autre par un des services sociaux de la ville, une copie du rapport est envoyée au service communal responsable de la bienfaisance. Cette pratique qui ne repose sur aucun texte législatif, permet d'exercer un fort contrôle social et d'introduire de nouveau, au moins encore partiellement, le point de vue belge qui repose plus sur des jugements moraux. Il semble bien que l'aide sociale soit utilisée dans bien d'autres domaines comme un régulateur pour 'discipliner' des couches de la population dont le comportement ne correspond pas aux attentes d'une certaine élite belge. Début janvier 1917, la police des mœurs allemande ne communique plus les listes des femmes mises sous contrôle au bureau des mœurs résiduaire à cause de ces 'abus'. Broché, le chef du bureau des mœurs, propose alors "de prévenir les divisions de nous signaler confidentiellement par une petite note, toute femme qui depuis cette date aurait été portée sur les contrôles " ${ }^{93}$. Je ne sais pas si cette proposition a été accueillie favorablement par Crespin et Lemonnier. Le bureau des mœurs semble s'être spécialisé pendant la guerre dans la lutte contre la prostitution des mineurs. La loi du 26 mai 1914 sur la traite des blanches n'a pas été abrogée par l'occupant. L'attitude des Allemands face à ce problème n'est pas très claire. L'article 10 de mars 1915 interdit aux prostituées d'engager une relation avec des mineurs. Par contre, aucune ligne n'est consacrée aux mineures qui se livrent à la prostitution. Elles sont cartées par les autorités allemandes et soumises comme les autres prostituées à des contrôles médicaux. Une fois sorties de l'hôpital Molière, elles sont parfois internées par décision des tribunaux des enfants dans des refuges jusqu'à leur majorité. Il ne ressort cependant pas des archives s'il s'agit là d'une pratique courante ou plutôt de cas isolés. La réorientation et l'activisme de Broché s'expliquent peut-être aussi par sa situation : il est le chef d'un service qui a perdu les trois quarts de ses collaborateurs et qui n'a plus de véritable raison d'être à ce moment.

À propos de ce sujet, il serait peut-être intéressant de soulever un problème méthodologique difficile à résoudre. La police n'est pas uniquement un instrument de pouvoir dans la main des entités communales, mais a aussi ses propres intérêts, ses buts autonomes. Il semble bien que lesdites autorités aient été prêtes à abandonner la surveillance de la prostitution à la police allemande (à part un contrôle indirect par les copies des rapports conservées à la Division Centrale). Or, la police bruxelloise aussi bien au niveau des divisions que dans la Division Centrale (bureau des mœurs) ne paraît pas prête à renoncer à cette tâche pour laquelle elle estime disposer d'une certaine compétence. Ainsi deux mois après avoir fixé l'attitude de la police face à la nouvelle réglementation, Lemonnier doit rappeler que «notre règlement sur le même sujet [la prostitution] est provisoirement inapplicable», "que l'Autorité Allemande s'est réservée le service des mœurs » et qu'il faut donc "agir avec modération pour éviter tout abus ». C'est peut-être faire montre de peu de prudence de conclure à partir de 
quelques indices à un conflit d'intérêt entre la police et l'autorité communale. Ce cas permet cependant de problématiser l'autonomie de ce corps relevé notamment par les travaux du sociologue français Monjardet et briser au moins momentanément notre discours d'historien qui part la plupart du temps du principe qu'il y a unité intrinsèque entre les deux organismes ${ }^{94}$.

Malgré les demandes de Lemonnier de mars 1915 de réduire le personnel et ainsi les coûts, les effectifs des deux services - la police des mœurs allemande ( 20 personnes) et l'hôpital Molière (71 personnes dont 24 religieuses) - restent les mêmes pendant toute la guerre. Les frais à payer pour le service médical sont multipliés par 1,7 à la fin de la guerre par rapport à 1915. À part les salaires, ce sont surtout les dépenses alimentaires qui, compte tenu de la difficulté de se procurer des denrées, expliquent cette hausse des contributions à payer par les 16 communes du Grand Bruxelles.

\section{b) Enregistrement et médicalisation des prostituées}

61 Les prostituées arrêtées sont le plus souvent dirigées au dépôt communal. Là " elles sont embrigadées, notées, classées » et semblent ainsi déjà devenir inoffensives si on en croit la description de l'Écho de la presse $e^{95}$.Le lendemain, elles sont transportées à l'hôpital Molière à Saint-Gilles où la deuxième étape du système de surveillance allemand entre en action. Comme au niveau de la surveillance policière, le contrôle médical est aussi centralisé pour le Grand Bruxelles. L'hôpital Molière avec une capacité de presque 300 lits, devient un lieu où les médecins ne traitent que des malades vénériennes. Ce service est dirigé en permanence par au moins deux médecins allemands dont toujours au moins une femme. Dans le contrôle de la prostitution, l'Allemagne, contrairement à la Belgique, utilisait déjà du personnel féminin au début $\mathrm{du} \mathrm{XX}^{\mathrm{e}}$ siècle. Les premières femmes dans la police bruxelloise vont d'ailleurs être engagées dans le service des mœurs pendant la Deuxième Guerre mondiale... à nouveau à l'instigation de l'occupant allemand ${ }^{96}$. Le premier chef de l'hôpital de Saint-Gilles est le docteur Frede déjà cité auparavant. Le poète allemand Gottfried Benn y aurait travaillé d'après son autobiographie. Cette expérience a d'ailleurs donné lieu à quelques écrits qui se caractérisent par le regard froid et le vocabulaire scientifique de Benn, mais qui sont les seules 'descriptions' dont on dispose sur cet hôpital ${ }^{97}$. Si le personnel supérieur est entièrement composé d'Allemands, les servantes et infirmiers sont presque tous des Belges; ceux-ci sont assistés par des franciscaines de Louvain dont la majorité serait d'origine allemande ${ }^{98}$. Une fois que la prostituée peut quitter l'hôpital, une assistante sociale allemande essaie d'assurer un suivi des patients et d'éviter un retour dans la prostitution. Cette mesure qui selon von Drigalski, Gouvernementsarzt, est particulièrement nécessaire en Belgique où des nombreuses prostituées sont poussées sur le trottoir à cause de la misère sociale; il ne s'agit donc pas nécessairement comme en temps de paix de personnes 'détériorées' ${ }^{99}$.

62 Le nombre des prostituées souffrant de maladies vénériennes est relativement important; les autorités allemandes y voient une justification des mesures qu'elles ont prises. Presque 55 \% des personnes placées sous contrôle médical le 1er mai 1915 sont atteintes d'une maladie vénérienne (Grand Bruxelles). L'efficacité du système allemand se révèle en 1917. Le pourcentage des prostituées malades placées sous contrôle médical est tombé à $40 \%$. 
Tableau 2 - Bilan médical de la prostitution pour le Brabant et Bruxelles pendant la première moitié de 1917100

\begin{tabular}{|l|l|l|l|l|l|l|l|l|}
\hline & $\begin{array}{l}\text { Prostituées } \\
\text { sous } \\
\text { contrôle }\end{array}$ & $\begin{array}{l}\text { Malade } \\
\text { en }\end{array}$ & danseuses & $\begin{array}{l}\text { Malade } \\
\text { en \% }\end{array}$ & $\begin{array}{l}\text { amenée par } \\
\text { la Sittenpolizei }\end{array}$ & $\begin{array}{l}\text { Malade } \\
\text { en \% }\end{array}$ & $\begin{array}{l}\text { Prostituées } \\
\text { amenées de } \\
\text { l'extérieur }\end{array}$ & $\begin{array}{l}\text { malade } \\
\text { en \% }\end{array}$ \\
\hline $1 / 1917$ & 5020 & 3,8 & 207 & 4,8 & 252 & 18,7 & 17 & 88,2 \\
\hline $2 / 1917$ & 4735 & 3 & 209 & 2,4 & 246 & 17,1 & 9 & 66,7 \\
\hline $3 / 1917$ & 5500 & 3 & 239 & 3,3 & 269 & 16,7 & 16 & 75 \\
\hline $4 / 1917$ & 4435 & 3,6 & 170 & 1,2 & 228 & 14,9 & 9 & 75 \\
\hline $5 / 1917$ & 4705 & 4,3 & 192 & 1 & 302 & 21,7 & 16 & 81,2 \\
\hline $6 / 1917$ & 4885 & $?$ & 207 & 3,4 & 310 & 23,3 & 13 & 84 \\
\hline
\end{tabular}

63 À Anvers, les chiffres sont légèrement inférieurs à ceux enregistrés pour Bruxelles. Dans l'imaginaire collectif, la syphilis joue un rôle important et semble être la maladie vénérienne par excellence. Elle est certes la plus dangereuse, mais à peine $4,5 \%$ des prostituées en sont atteintes, la très grande majorité étant touchée par la gonorrhée.

La raison principale d'améliorer la police des mœurs est d'ordre militaire, la peur qu'une partie des soldats ne soient immobilisés par des maladies vénériennes. Sans pouvoir et vouloir analyser cette problématique, j'ai cependant jugé utile de présenter quelques chiffres qui ne sont pas forcément représentatifs, mais qui permettent au moins de se faire une idée de l'incidence des maladies sexuellement transmissibles sur le front de l'ouest. Un huitième des soldats de la 6e armée allemande qui se retrouvent à l'hôpital en février-mars 1917, sont atteints de maladies vénériennes. Ces dernières représentent la troisième maladie (derrière la grippe et la pneumonie) et touchent un peu plus d'un pour cent des soldats ${ }^{101}$. Les troupes d'occupation sont aussi astreintes à des contrôles réguliers. Une fois par semaine, les soldats sont soumis à un contrôle médical. Avant de pouvoir rentrer en Allemagne, chaque homme doit se faire examiner. Les autorités militaires ne sont pas gênées par une pudeur quelconque. De véritables cours d'éducation sexuelle sont organisés. Des consultations qui permettent de garantir le plus possible l'anonymat, sont mises en place. À côté d'une prévention sanitaire, les autorités médicales proposent de dévier l'instinct sexuel vers des activités sportives. C'est l'absence de pudeur qui choque les Belges. Charles Gheude, membre de la députation permanente à la province du Brabant pendant la Première Guerre mondiale, s'offusque des affiches qui prônent l'utilisation du préservatif, mot qu'il refuse même d'écrire : «Je lis : 'En vue de prévenir encore davantage la communication des maladies sexuelles, des pr...' Diable ! comment continuer (...) Ils en mettent en vente; ils en ont le monopole vis-à-vis de leurs soldats. Non seulement ils conduisent ceux-ci en groupes vers les asiles de l'amour, mais encore ils les munissent ou munissent celles qui attendent, de ... 'Un mark, la pièce; c'est le prix de revient', annonce le signataire de l'avis, lequel n'est autre que le Président de l'administration civile allemande, chef de la police des mœurs pour le Grand Bruxelles!'Il est instamment recommandé de se procurer... (l'objet en question) et de prendre soin qu'il soit employé à chaque'... (et le 
mot suit - tout cru !). À côté, à titre d'annexe, le détail, précis et cynique, des mesures à prendre par les deux sexes - avant, après - pour parer au coup de pied de Vénus. C'est plat, vulgaire, écœurant... $»^{102}$. Cette politique 'vulgaire' porte cependant ses fruits : le pourcentage de soldats atteints de maladies vénériennes parmi les troupes d'occupation est plus bas que sur le front : entre août 1916 et juillet $191740 \%$ o sont touchés par une maladie vénérienne ${ }^{103}$.

Mais derrière ce souci en premier lieu militaire se cache une peur plus profonde. À partir de la fin du XIXe siècle, les maladies vénériennes se voient accorder une place prépondérante dans les discussions démographique et eugénique qui battent leur plein en Europe. Plus spécifiquement la syphilis est considérée comme une menace pour la 'santé raciale' de la nation. Pendant la Première Guerre mondiale, dans un moment où le corps national (dans les deux sens du mot) est particulièrement mis à l'épreuve, ces maladies sont d'autant plus dangereuses ${ }^{104}$. Lors de son discours d'introduction à la conférence d'octobre 1915, von Bissing parle de ces maladies « qui rongent la moelle de notre force nationale $»^{105}$. Presque tous les orateurs reviennent sur ce sujet : sauver la force de la race germanique par une lutte énergique contre la prolifération des maladies vénériennes. Le discours est marqué par un vocabulaire biologique : un autre auteur parle du danger pour les cellules du corps national (Volkskörper).

\section{Une démobilisation difficile à mettre en place ${ }^{106}$}

\section{a) Une épuration qui marque le corps}

À Bruxelles, la Première Guerre mondiale se termine dans une atmosphère révolutionnaire. Pendant les derniers jours de l'occupation, ce ne sont plus les structures mises en place en 1914 qui dirigent Bruxelles. C'est un conseil des soldats. Le 17 novembre, pendant que les derniers soldats quittent la ville, Max y fait sa 'joyeuse entrée'. Celle-ci est en partie ternie par des explosions dans différentes gares. Des trains délaissés par les Allemands sont pillés par des milliers de Bruxellois. Mais parmi les denrées alimentaires et autres produits se trouvent aussi des munitions dont l'explosion provoque la mort de plusieurs personnes. Pendant plusieurs heures la police bruxelloise est incapable de rétablir l'ordre. Désarmée depuis le début de la guerre, elle est confrontée à des groupes qui possèdent toutes sortes d'armes. Mais ce n'est pas le seul problème. Si de nombreux activistes flamingants quittent la ville avec les Allemands en direction des Pays-Bas et de l'Allemagne, des rassemblements tumultueux ont lieu jusqu'au 24 novembre devant des maisons de collaborateurs et d'Allemands.

Dans la plupart des contributions sur la libération pendant la Deuxième Guerre mondiale, la tonte de femmes est devenue un sujet incontournable. Dans un récent ouvrage consacré à ce sujet en France, l'auteur souligne le caractère presque anthropologique du phénomène en remontant à la persécution des sorcières au XVI ${ }^{\mathrm{e}}$ siècle, mais ne relève pas si de tels cas ont été observés en $1918^{107}$. Le 21 novembre 1918 le docteur Bayet note les mots suivants dans son journal personnel: "On arrête à Bruxelles les activistes, les traîtres, les accapareurs. On casse les maisons de ceux qui ont manifesté des sentiments germanophiles; enfin les femmes qui ont eu des rapports avec les Allemands sont rasées, mises en chemises et promenées dans la rue sur des chariots $»^{108}$. 
Comme nous l'avons vu auparavant, les premières semaines de l'occupation se caractérisent par plusieurs rassemblements devant les maisons de débauche fréquentées par les soldats allemands. Une fois cette colère apaisée, ces actes deviennent plus rares, mais ne disparaissent jamais pendant toute la guerre. Ainsi deux femmes qui accompagnent deux soldats dans l'exposition au Luna Park sont injuriées en été 1916; lorsqu'elles s'arrêtent pour répondre aux accusations, elles sont vite entourées de 200 personnes qui les suivent en les huant et sifflant et la police doit intervenir ${ }^{109}$. De même, pendant toute la guerre des lettres anonymes dénoncent auprès des autorités communales l'attitude de certaines femmes s'étant engagées avec des soldats allemands. Leurs auteurs ne voient pas nécessairement la différence entre les prostituées et les femmes qui vivent une histoire d'amour avec un Allemand. Il semble impossible qu'une femme s'engage avec un Allemand sans en tirer des avantages. En 1918 ces sentiments peuvent s'exprimer librement. Les forces de l'ordre ont d'autres chats à fouetter. La dénonciation anonyme même si elle existe en temps de paix, semble avoir connu un développement exceptionnel pendant la guerre. Même si von Bissing déclare dans un avis en mai 1915 qu'il refuse «de donner une suite quelconque à de telles dénonciations $»^{110}$, tant la police belge qu'allemande semblent jusqu'à la fin de la guerre vérifier la plupart des informations qu'elles colportent.

Le fait que Bayet qui en l'espace de quatre ans noircit 6353 pages n'exprime pas son étonnement devant la tonte des femmes est un indice qu'il s'attend à cette réaction. Même si nous ne pouvons pas jauger l'étendue du phénomène, Bayet nous livre deux éléments formels importants : il s'agit bien d'une punition corporelle et publique. Le 23 novembre 1918, plusieurs centaines de personnes sont témoins d'une tonte au centre de Bruxelles. Une réfugiée est accusée par un autre réfugié d'avoir «couru avec des boches » et d'avoir " trahi son père ». Il fait appel à des soldats pour l'arrêter. Très vite, une foule se rassemble autour d'elle. On commence par lui arracher son chapeau; une fois ce premier acte accompli, tout va très vite. Elle est dénudée et ses cheveux sont coupés. Ce n'est cependant pas la population du quartier, mais des soldats belges, français et anglais qui accomplissent cet acte. Jusqu'à la fin de ce charivari, ce sont toujours des soldats qui posent les gestes de violence. Est-ce dû à une brutalisation suite à l'expérience du front ou faut-il plutôt relever l'hypothèse de Fabrice Virgili selon laquelle ces actes sont toujours effectués par des personnes étrangères au contexte local ? Une fois le corps marqué, la femme, nue, est traînée sur les escaliers de la Bourse. À six heures du soir, il s'agit d'un des lieux les plus fréquentés de Bruxelles. Là-bas, elle est victime de coups et d'attouchements sexuels. D'après un rapport de police, les agents ne peuvent pas (ne veulent pas?) la libérer; finalement d'autres soldats interviennent et la conduisent au commissariat. Dans la voiture, elle est de nouveau victime d'attouchements sexuels. Un consul honoraire de Belgique qui est témoin de cette scène, ne met pas en question la punition en soi. Il se plaint que des "centaines d'enfants" ont été témoins de la scène; les hommes ont commis "un outrage public à la pudeur » et cédé «à une impulsion de délire érotique ». De tels actes risquent de provoquer de mauvaises impressions chez «les hôtes étrangers » et risquent de « compromettre la moralité publique $»^{111}$.

70 Les deux éléments-clés relevés par Bayet se retrouvent aussi dans cet exemple. L'épuration est sexuée. Contrairement à la punition d'un collaborateur, celle d'une 'collaboratrice' s'inscrit souvent sur le corps, un corps avec lequel elle a 'trahi' la patrie. En coupant les cheveux de la femme, les auteurs lui enlèvent symboliquement sa 
féminité. Dans notre cas, ce sont uniquement des hommes qui exercent leur violence sur un corps de femme. Ensuite, la punition se déroule devant le plus large public possible, la Bourse étant un des lieux publics par excellence à Bruxelles. Comme la 'trahison' avait été publique, il s'agit maintenant de laver l'affront devant tout le monde.

\section{b) Une deuxième occupation?}

Pendant quelques mois, Bruxelles va vivre une 'deuxième occupation'. La ville continue d'héberger un nombre important de soldats et la prostitution reste un sujet épineux. Avant même le départ définitif des derniers soldats allemands, le procureur du Roi Holvoet exprime ses craintes "sur la répercussion que pourrait avoir sur la santé publique l'arrêt brusque du service des mœurs de l'Autorité allemande ». Il requiert le Bourgmestre de Saint-Gilles pour assurer le service à l'hôpital local. Le lendemain, le même sujet est traité à la conférence des Bourgmestres qui siège à ce moment tous les jours ${ }^{12}$. Aussi bien au niveau national que communal, toutes les autorités soulignent l'utilité de garder la structure centralisatrice introduite par l'occupant. Mais, dès le départ de la police allemande, chaque police communale retrouve son autonomie. Pendant plusieurs années, la conférence des Bourgmestres essaie de maintenir un service médical intercommunal. La commune de Saint-Gilles refuse cependant de mettre son hôpital plus longtemps à la disposition et libère en mars 1919 toutes les prostituées qui s'y trouvent, provoquant ainsi un scandale retentissant. Six mois plus tard un hôpital intercommunal vénérien s'ouvre à Uccle. Dès 1921, des voix s'élèvent contre " cet hôpital [qui] est en réalité une prison où ne sont enfermées que les femmes soumises au contrôle $»^{113}$. En 1924, la Ville de Bruxelles tente une expérience originale : elle abolit provisoirement le règlement sur la prostitution. L'échevin et futur bourgmestre Van de Meulebroeck dira que la « réglementation de la prostitution étant supprimée, il n'y a donc plus de prostituées (sic) et nous ne pouvons plus envoyer les filles publiques malades dans un hôpital spécial; l'hôpital actuel n'est donc plus nécessaire ${ }^{114}$. Exit l'hôpital à Uccle, qui sera fermé la même année.

Si les structures mises en place ne survivent pas très longtemps au départ de leurs initiateurs, les problèmes semblent en partie rester les mêmes. La cocaïne continue à circuler parmi les prostituées. Après les premières joies de la libération, la présence des soldats commence vite à être ressentie comme une charge. Ainsi la rue Neuve qui était un lieu de 'rencontres' entre 1914 et 1918, ne change pas de fonction comme en témoigne cette lettre anonyme qui est un exemple parmi plusieurs : «Je passais hier (...) dans la rue Neuve. Pour la première fois de ma vie, j’ai été révolté par le spectacle qu'y donnaient des soldats américains. Le moindre était de voir des hommes de trente à quarante ans au bras de fillettes de treize à quatorze ans. On appliqua l'épithète des 'cochons' au nom des boches, mais jamais je n'ai vu ceux-ci se permettre la dixième partie de ce que se permettent les Américains ${ }^{115}$. Mais la sortie de guerre difficile est une autre histoire...

\section{Conclusions}

L'occupation fut-elle une rupture dans la pratique prostitutionnelle? 
74 La présence de milliers de soldats à Bruxelles bouleversera profondément ce milieu. Certes, il semble y avoir continuité dans la répartition spatiale des prostituées : ce sont les lieux d'avant-guerre qui sont (ré)investis pendant le conflit. Mais le nombre de femmes qui se prostituent explose. Bruxelles est à la fois le siège d'une importante administration allemande et un lieu de passage et de repos pour les troupes luttant sur le front de l'ouest. Elle devient la 'succursale de Paris' avec tout ce que cette métaphore comporte d'imaginaire et de réalité. Cette explosion du nombre de prostituées - la comparaison avec l'avant-guerre en chiffres réels étant impossible vu que le contrôle est beaucoup plus renforcé pendant l'occupation - fait monter la question d'où surgissent ces femmes. La plus grande partie de ces nouvelles prostituées viennent de l'agglomération bruxelloise. Pour de nombreuses femmes, la Première Guerre mondiale constitue donc une entrée provisoire dans cet univers. Comment cette expérience a-telle été vécue ? Comment se sont-elles de nouveau réintégrées dans la société après la guerre, lorsque le nombre de prostituées diminue fortement après le départ des soldats alliés? Ces questions restent sans réponse encore aujourd'hui. Les cas de tonte relevés ainsi que les études consacrées à ce sujet montrent l'opprobre qui pèse sur ces femmes qui sont doublement marginalisées : par leur corps elles auraient trahi et la morale et leur nation. La prostituée est subversive sur les deux plans. D'ailleurs, après la guerre, toutes les femmes de plus de 21 ans, reçoivent le droit de vote au niveau communal, à part les prostituées ${ }^{116}$.

75 L'occupation fut-elle une rupture dans l'histoire du contrôle de la prostitution à Bruxelles?

76 La présence des Allemands provoque non seulement une demande de prostituées plus importante, mais le contrôle de celles-ci sera aussi fortement renforcé. Après quelques mois d'hésitation, les autorités occupantes instaurent un système de surveillance étroit. La politique allemande concernant la prostitution se caractérise par son côté fonctionnaliste. Les autorités allemandes essaient à travers cette surveillance de diminuer les maladies sexuellement transmissibles. Celles-ci peuvent exténuer le Volk sur le front extérieur - affaiblissement de l'armée allemande dans sa lutte contre les Alliés dans les tranchées - et sur le front intérieur - garantir la santé du corps national. Leur action préventive ne s'adresse pas seulement aux femmes, mais aussi à la population masculine (allemande). Le contrôle de la prostitution n'est plus seulement sexué. La surveillance des soldats est d'ailleurs aussi assez contraignante : le contrôle hebdomadaire des militaires est aussi lourd que celui des prostituées. La prostitution en tant que telle n'est pas mise en question. Il ne s'agit même pas d'un mal nécessaire comme l'avait encore définie Parent-Duchâtelet; bien au contraire quelques hauts fonctionnaires n'hésitent pas à lui reconnaître un effet thérapeutique (faire oublier les expériences du front). L'armée belge sur le front d'Yser semble mener une politique plus pudique; la prostitution y est un 'non-sujet'117.

Par contre pour les autorités belges, il ne s'agit pas en premier lieu d'une lutte contre les maladies sexuellement transmissibles mais contre la prostitution elle-même. Leur approche intentionnaliste est un mélange entre réglementarisme - inventorier et ainsi contrôler les prostituées - et abolitionnisme - éradication à long terme de la prostitution. Si la politique centralisatrice, instaurée par les Allemands, est probablement reconnue comme utile et constitue une poussée modernisatrice (uniquement à court terme), l'approche fonctionnaliste ne rencontre aucune compréhension. La nécessité du devoir patriotique en temps d'occupation n'offre pas 
d'espace pour la prostituée. Le corps de police affiche une attitude ambiguë : d'une part il y a ceux qui travaillent dans le cadre instauré par l'occupant et semblent remplir leur tâche à la satisfaction de celui-ci; d'autre part, il y a ceux qui sont exclus des nouvelles structures et qui refusent d'abandonner une partie de leurs compétences.

Le contrôle de la prostitution comme test case d'une coopération entre occupé et occupant?

Si les approches sont donc différentes, la réalité sur le terrain est seulement marquée de tensions au moment de la mise en place du système de surveillance. La Sittenpolizei a recours à des agents belges qui opèrent sous sa direction sans que cela provoque une réaction particulière de la part des autorités communales. Est-ce que cette absence de conflits à long terme s'explique par le fait que le contrôle touche un groupe de femmes marginalisées aussi bien par l'occupant que par l'occupé ? Certes, il semble bien que la coopération policière n'ait été dans aucun autre domaine aussi loin que dans celui du contrôle prostitutionnel. Mais malgré de nombreux heurts entre les bourgmestres successifs de Bruxelles et les autorités allemandes, le maintien de l'ordre ne devient jamais un point de rupture ${ }^{118}$. Par contre, quelques réformes allemandes (comme la centralisation) prônées avant la guerre par une partie du monde politique auront un effet pervers dans l'après-guerre: elles seront abolies non parce qu'elles étaient mauvaises, mais parce qu'introduites par l'occupant.

\section{BIBLIOGRAPHIE}

Becker, A., Oubliés de la Grande Guerre, Paris, Noêsis, 1998.

Benn, G., Der Vermessungsdienst. Erkenntnistheoretisches Drama, Aktionsbücher der Aeternisten, 1919, 9, pp. 36-60.

Benn, G., Epilog, Die Zukunft 30, 1921, 115, pp. 57-61.

Berlière, J.-M., La police des mœurs sous la IIIe République, Paris, Seuil, 1992.

Bulletin communal de la Ville de Bruxelles, Bruxelles, Ville de Bruxelles.

Corbin, A., Les filles de noce. Misère sexuelle et prostitution au XIX ${ }^{e}$ siècle, Paris, Champs Flammarion, 1978, 1995.

Delcourt, F., Encyclopédie des fonctions de police, 1er vol., Tournai, Imprimerie Vasseur-

Delmée, 1905.

Delplancq, T., Des civils dans la guerre. Une approche du sort des évacués français en Belgique, 1917-1918, inLiber amicorum Jacques-Henri Lefebvre, La Louvière, Ceredore, 2001, pp. 71-80.

De Koster, M., Ongepast gedrag van jonge vrouwen en (generatie) conflicten. Ouderlijke klachten over onhandelbare dochters bij de kinderrechter van Antwerpen in 1912-1913 en 1924-1925, Cahiers d'Histoire du Temps Présent, 2001, 8, pp. 101-131. 
De Schaepdrijver, S., De Zonde in Banen geleid : gereglementeerde prostitutie in Brussel 1844-1877. Onderzoek naar houd(st)ers van getolereerde bordelen, mémoire de licence présenté à la VUB, Bruxelles, 1983.

De Schaepdrijver, S., 'Filles au fait du langage du monde' : gereglementeerde prostitutie in Brussel (1844-1877) als indicator voor een sociolinguistische segregatie ?, Taal en sociale integratie, 1984, 7, pp. 3-51.

De Schaepdrijver, S., Regulering van prostitutie in de negentiende eeuw. Een concreet voorbeeld : Brussel, 1844-1877, inL'initiative publique des communes en Belgique 1795-1940, vol. 2, Bruxelles, 1986, pp. 537-558.

De Schaepdrijver, S., La Belgique entre exaltation et rejet, 1914-1918, Cahiers d'Histoire du Temps Présent, 2000, 8, pp. 17-49.

Dupont-Bouchat, M.-S., La prostitution urbaine. La marginalité intégrée, in Gubin, E., Nandrin, J.P. (Eds), La ville et les femmes en Belgique. Histoire et sociologie, Bruxelles, Facultés universitaires Saint-Louis, 1993, pp. 97-129.

Dupont-Bouchat, M.-S., Verdraagzaamheid en repressie. Fascinatie en weerzin. Elkaar dwarsende blikken op de prostitutie in België (15de-20ste eeuw, inVan badhuis tot eroscentrum. Prostitutie en vrouwenhandel van de middeleeuwen tot heden, Bruxelles, Archives Générales du Royaume, 1996, pp. 51-87.

Fisher, C.H., La vie érotique pendant la guerre, traduit et adapté par Jacques Gorville, Paris, Édition Internationale Aldor, s.d.

Föllmer, M., Die bürgerliche Ordnung der Nation. Zur Diskursgeschichte von Industriellen und hohen Beamten in Deutschland und Frankreich 1900-1930, thèse remise à la Humboldt-Universität, Berlin, 2000.

Gesetz- und Verordnungsblatt für die okkupierten Gebiete Belgiens.

Gheude, C., Nos années terribles 1914-1918. Tome troisième - La libération, Bruxelles, Oscar Lamberty, 1919.

Gubin, E., Bespiegelingen over sekse en oorlog in België, 1914-1918, Jaarboek voor Vrouwengeschiedenis, 1995, 15, pp. 33-48.

Halasi, O., Belgium under the German Heel, London, New York, Toronto and Melbourne, Cassell, 1917.

Hirschfeld, M., Gaspar, A., Sittengeschichte des Ersten Weltkrieges, Hanau, Komet, 1929, 1994.

Horne, J., Introduction, Démobilisations culturelles après la Grande Guerre. 14-18 - Aujourd'hui - Today Heute, 2002, 5, pp. 43-53.

Huberty, C., Réglementarisme et abolitionnisme en matière de prostitution à Bruxelles (1830-1914), mémoire de licence présenté à l'ULB, Bruxelles, 1982.

Huberty, C., Keunings, L., La prostitution à Bruxelles au dix-neuvième siècle, Les cahiers de la Fonderie, 1987, 2, pp. 3-21.

Janssens, P., Belgische Veldhospitalen tijdens de Eerste Wereldoorlog, Bruxelles, Archives Générales du Royaume, 2001.

Keunings, L., Histoire de la police à Bruxelles (1831-1914), mémoire de licence présenté à l'ULB, Bruxelles, 1980 
Köhler, L., Die Staatsverwaltung der besetzten Gebiete. Erster Band Belgien, Stuttgart - New Haven, Deutsche Verlagsanstalt, 1927.

Laquer, B., Ethisches im Kampf gegen die Volkskrankheiten, Therapie der Gegenwart, avril 1917, pp. $1-2$.

Le Naour, J.-Y., Femmes tondues et répression des 'femmes à boches' en 1918', Revue d'histoire moderne et contemporaine, janvier-mars 2000, pp. 148-158.

Le Naour, J.-Y., Misères et tourments de la chair durant la Grande Guerre, Paris, Aubier, 2002.

Liepmann, M., Krieg und Kriminalität in Deutschland, Stuttgart, Deutsche Verlagsanstalt, 1930.

Liulevicius, V.G., War Land on the Eastern Front. Culture, National Identity and German Occupation in World War I, Cambridge, Cambrige University Press, 2000.

Lorenz, M., Leibhaftige Vergangenheit. Einführung in die Körpergeschichte, Tübingen, edition diskord, 2000 .

Majerus, B., Controlling urban society in Brussels during World War I, in Chickering, R., Funck, M. (Eds), Endangered Cities: Military Power and Urban Society in the Era of the

World Wars, Boston, Brill, à paraître en 2003.

Massnahmen zur Bekämpfung der Geschlechtskrankheiten. Besprechung im Sitzungssaal des GeneralGouvernements in Belgien, s.d.

Meinen, I., Wehrmacht und Prostitution im besetzten Frankreich, Bremen, Edition Temmen, 2002.

Mertens, P., Les éblouissements, Paris, Seuil, 1987, 1998.

Monjardet, D., Ce que fait la police. Sociologie de la force publique, Paris, éditions La découverte, 1996.

Nienhaus, U., Einsatz für die 'Sittlichkeit' : Die Anfänge der weiblichen Polizei im

Wilhelminischen Kaiserreich und in der Weimarer Republik, in Lüdtke, A. (Ed.), 'Sicherheit' und 'Wohlfahrt' Polizei, Gesellschaft und Herrschaft im 19. und 20. Jahrhundert, Frankfurt a.M., Suhrkamp, 1992, pp. 243-266.

Racine, A., La délinquance de la jeunesse en Belgique en temps de guerre, Revue internationale de l'enfant, 1944, 8, pp. 1-8.

Reinke, H., Die Polizei und die 'Reinhaltung der Gegend'. Prostitution und Sittenpolizei im Wuppertal im 19. und im frühen 20. Jahrhundert, in Reulecke, J., Castell Rüdenhausen, A. (Eds), Stadt und Gesundheit. Zum Wandel von 'Volksgesundheit' und kommunaler Gesundheitspolitik im 19. und frühen 20. Jahrhundert, Stuttgart, Franz Steiner Verlag, 1991, pp. 129-143.

Rüdiger, U., Otto Dix. Gemälde, Zeichnungen, Druckgrafik, Munich, Klinkhardt \& Biermann, 1997.

Schwerhoff, G., Einführung in die Historische Kriminalitätsforschung, Tübingen, edition diskord, 1999.

Schubert, D., Otto Dix, Reinbek bei Hamburg, Rororo, 1980, 200.

Un souvenir historique. Les avis, proclamations \& nouvelles de guerre allemandes affichés à Bruxelles pendant l'occupation, 35 vol., Bruxelles, Ed. Brian Hill, s.d.

Vierset, A., Mes souvenirs. Sur l'occupation allemande en Belgique, Paris, Librairie Plon, 1932.

Virgili, F., La France 'virile’. Des femmes tondues à la libération, Paris, Payot, 2000. 


\section{NOTES}

1. L'écriture de cette contribution a été accompagnée par plusieurs personnes qui, par leurs compétences respectives, l'ont fortement enrichie. Les discussions ont montré qu'elles ne partagent pas nécessairement toutes mes conclusions, en d'autres termes, elles ne pourraient pas être tenues responsables pour les fautes et interprétations qui s'y trouvent. Qu'Alain Colignon, Sophie De Schaepdrijver, Marie-Sylvie Dupont-Bouchat, Chantal Kesteloot, Tonia Theisen ainsi que les trois lecteurs de Crime, Histoire \& Sociétés soient vivement remerciés pour leur aide.

2. Dix, Otto, Frontsoldat in Brüssel, gravure, 1924, 28,8 * 19,8 cm (Inv. Nr. D/G 87), dans Rüdiger (1997, p. 212).

3. "Ich wollte die Dinge zeigen, wie sie wirklich sind », cité d'après Schubert (2001). Toutes les informations sur la vie de Dix proviennent de cette monographie.

4. Meinen (2002), Les informations contenues dans le livre de Jean-Yves Le Naour sur les départements français occupés pendant la Première Guerre mondiale ont plutôt un caractère anecdotique: Le Naour (2002, plus particulièrement les pp. 142-146 et pp. 161-162). Sur l'historiographie des territoires occupés toujours lacunaire : Becker (1998, pp. 13-20).

5. Pour l'historiographie européenne je me limiterai à deux ouvrages: Schwerhoff (1999, plus particulièrement pp. 156-160) et Lorenz (2000) ainsi qu'à la bibliographie suivante: http :// www.univie.ac.at/Wirtschaftsgeschichte/sexbibl/; pour la Belgique : Keunings (1980), Huberty (1982) et De Schaepdrijver (1983). Marie-Sylvie Dupont-Bouchat est la dernière à s'être intéressée de plus près à cette question $(1993 ; 1996)$.

6. Pasinomie, 1836, p. 131; pour plus de renseignements sur le cadre législatif : Delcourt (1905, pp. 430-434).

7. Corbin (1995, pp. 24-25).

8. Huberty (1982, p. 57).

9. Berlière (1992, pp. 55-69).

10. Huberty/Keunings (1987, p. 20).

11. À la veille de la Première Guerre mondiale le ‘Grand Bruxelles' est composé des communes de Bruxelles, d'Anderlecht, d'Auderghem, d'Etterbeek, de Forest, d'Ixelles, de Jette-Saint-Pierre, de Koekelberg, de Laeken, de Molenbeek, de Saint-Gilles, de Saint-Josse-ten-Noode, de Schaerbeek, d'Uccle, de Watermael-Boitsfort et de Woluwe Saint Lambert.

12. Rapport intitulé 'Prostitution - Ville de Bruxelles et communes faisant partie de la Conférence', non daté (probablement 1910); C(abinet du) B(ourgmestre) 544, A(rchives de la) $\mathrm{V}(\mathrm{ille}$ de) B(ruxelles).

13. Circulaire aux autorités communales du 4 août 1914 par Paul Berryer, ministre de l'Intérieur; Guerre 1914-1918, Archives de la Police, Fonds administratif (Pol14-18), AVB.

14. Vierset (1932, p. 61). Auguste Vierset est directeur du cabinet du bourgmestre de Bruxelles au moment de l'invasion allemande.

15. La plus grande partie de la Belgique se retrouve dans le Generalgouvernement. Les régions longeant le front sont regroupées dans l'Etappengebiet, une structure administrative dépendant seulement des corps d'armée. Le régime d'occupation y est souvent plus dur. La Belgique connaîtra trois gouverneurs généraux: Colmar von der Goltz (1843-1916) du 26 août au 2 décembre 1914, Moritz Freiherr von Bissing (1844-1917) du 2 décembre 1914 au 18 avril 1917 et Ludwig Freiherr von Falkenhausen (1844-1936) du 3 mai 1917 jusqu'à la fin de la guerre. Le gouverneur général qui dépend directement de l'Empereur, chapeaute l'administration allemande et se fait assister pour la gestion civile par un Verwaltungschef. Ce dernier poste est occupé d'août 1914 jusqu'en juillet 1917 par Max von Sandt. À ce moment cette position est doublée : en Flandre il sera repris par Schaible (1870-1933), en Wallonie par Haniel. La même structure - chef militaire secondé par un administrateur civil - se retrouve au niveau régional (Militärgouverneur - Präsident der Zivilverwaltung) et local (Kreischef - Zivilkommissar); Köhler (1927). 
16. 'Proclamation du Baron von der Goltz du 2 septembre 1914', Un souvenir... (tome I, pp. 7-8).

17. Note du 28 août 1914 par Adolphe Max; Pol14-18 - 519, AVB.

18. Note intitulée 'Diminution du personnel' (non datée); CB 803, AVB.

19. Questionnaire sur le service de la prostitution en vue de l'application des mesures prophylactiques contre les maladies vénériennes (non daté; probablement de janvier-février 1915); Pol14-18 - 420, AVB.

20. 'Halbjahresbericht für die Zeit vom 1. Februar bis 31. Juli 1917 - Gouvernement von Brüssel und Brabant'; H(andschriften-) S(ammlung) 2261, Bay(erisches) $\mathrm{H}$ (aupt)St(aats)A(rchiv) et 'Verwaltungsbericht des Verwaltungschefs bei dem Generalgouverneur in Belgien für die Zeit vom August-Oktober 1915', Rep. 89 (Zivilkabinett) H.-32457; Geh(eimes) St(aats)arch(iv) $\mathrm{P}$ (reussischer) K(ulturbesitz).

21. Extrait de la séance du conseil communal du 26 août 1914; CB 798, AVB.

22. Rapport de police du 21 octobre 1914 par la 2e division; Pol14-18 - 463 pour le premier cas et rapport de police du 24 septembre 1914 par la 2e division; Pol14-18 - 500, AVB pour le deuxième cas.

23. L'échevin Maurice Lemonnier (1860-1930) remplace Max comme bourgmestre lorsque ce dernier est déporté par les Allemands en septembre 1914. Lemonnier subit le même sort en mai 1917 et sera remplacé par un autre échevin, Louis Steens (1849-1933).

24. Rapport de police du 1er octobre 1914 par un commissaire adjoint de la 3e division au bourgmestre; Pol14-18 - 414, AVB.

25. Lettre du 21 septembre 1914 par les habitants de la rue aux Fleurs au bourgmestre; Pol14-18 414, AVB; souligné par l'auteur.

26. De Schaepdrijver (2000, pp. 22-23).

27. Meinen (2002, pp. 194-211). Liulevicius relève cette pratique sur le front de l'Est pendant la Première Guerre mondiale : Liulevicius (2000, p. 80).

28. Rapport du 12 novembre 1914 par le bureau des mœurs; Pol14-18 - 420, AVB.

29. Lettre du 12 novembre 1914 par Lemonnier à Bayer, commandant de la Kommandantur à Bruxelles jusqu'en février 1915 et télégramme $N^{\circ} 3000$ du 7 décembre 1914 par Lemonnier à toutes les divisions; Pol14-18 - 420, AVB.

30. Ernst Vogt, Erinnerungen aus Brüssels letzten Besatzungstagen Juli bis November 1918 (manuscrit); HS-2154, BayHStA. Ernst Vogt travaille dans la flamenpolitische Abteilung.

31. Edmond Crespin est commissaire de la Division Centrale en 1914. À ce moment, Bruxelles ne dispose pas de commissaire en chef, fonction remplie par le bourgmestre. Les autorités allemandes qui veulent un responsable pour traiter des problèmes du maintien de l'ordre pour le Grand Bruxelles, font de lui, de manière officieuse, une sorte de commissaire en chef pour les 16 communes. Cette position privilégiée lui vaut d'ailleurs après la guerre une information judiciaire qui se termine sur une ordonnance de non-lieu en sa faveur par l'Auditeur Militaire du Grand Quartier Général. En 1920, Crespin est nommé commissaire en chef de la ville de Bruxelles. 32. Gesetz... (13 février 1915, pp. 159-161).

33. Lettre du 14 janvier 1915 par Gerstein à Lemonnier; CB 762, AVB.

34. Lettre du 22 février 1915 par Freiherr v. Schoen à Bethmann-Hollweg et la réponse du 7 mars 1915 par Bissing; R1501 (Reichsministerium des Innern)-19345, B(undes)arch(iv) B(erlin) et « dass Frontoffiziere sich von den schweren seelischen und körperlichen Eindrücken (...) durch den Genuss harmloser Zerstreuungen in Brüssel rasch zu erholten pflegten und dass eine zu weit gehende Beschränkung in dieser Hinsicht eher schädlich als nützlich wirke », extrait d'une lettre du 10 février 1917 par von Bissing à Hurt; HS-2260, BayHStA. Quant à l'auteur de cette lettre, il pourrait s'agir de l'ancien ambassadeur d'Allemagne en France. Cette politique - la prostitution comme 'remède' contre «l'expérience dépressive des tranchées »- est déjà relevée dans la littérature contemporaine, e.a. par Liepmann (1930, p. 152). 
35. Lettre du 1er février 1915 par von Sandt au secrétaire d'État de l'Intérieur; R1501-19374, BarchB.

36. 'Ordonnance de police du 13 février 1915 par Gerstein', Un souvenir ... (tome III, p. 49).

37. Lettre du 27 février 1915 par Gerstein (destinataire inconnu); Pol14-18 - 420, AVB.

38. Lieux et dates de création des différents services de la police des mœurs: Bruxelles le 3 février 1915; Charleroi le 6 mars 1915 (agrandi le 20 juin 1917); Liège le 6 mars 1915; Namur le 6 mars 1915; Anvers le 6 mars 1915; Mons le 6 mars 1915; Thuin le 21 février 1918; Verviers le 23 mars 1918; Malines le 27 avril 1918; La Louvière le 29 juin 1918; Hal le 1er août 1918 (dépendance de Bruxelles).

39. Polizeiliche Vorschriften zur Sicherung der Gesundheit, der öffentlichen Ordnung und des öffentlichen Anstandes vom 9. März 1915; T. 506-17 (Archives de l'administration de la province du Brabant), A(rchives) G(énérales du) R(oyaume).

40. Berliner Tageblatt du 27 mars 1915; R1501-19376, BarchB; Reinke (1991, pp. 129-132).

41. «Während sie [die Sittenpolizei] in Antwerpen lebhafte Anerkennung fand, weigern sich einige Grossbrüsseler Gemeinden, $\mathrm{zu}$ den Kosten pflichtmässig beizutragen », Verwaltungsbericht des Verwaltungschefs bei dem Generalgouverneur in Belgien für die Zeit vom Mai - Juli 1915, p. 13; R1501-19455, BarchB.

42. Lettre du 6 mars 1915 par Lemonnier à Gerstein; Pol14-18 - 420, AVB; le règlement prévoit cependant une autorité de recours.

43. Lettre du 10 août 1915 par Gerstein à Lemonnier; Pol14-18 - 420, AVB.

44. Lettre du 4 mai 1915 par Lemonnier à Gerstein; Pol14-18 - 420, AVB.

45. Questionnaire sur le service de la prostitution...; Pol14-18 - 420, AVB.

46. Rapport du 29 juin 1915 par la 2e division; Pol14-18 - 420, AVB.

47. Note du 19 février 1915 par le bureau des mœurs; Pol14-18 - 420, AVB.

48. Massnahmen..., (s.d., p. 5).

49. Laquer (1917).

50. Les thèses et projets défendus par certains militaires lors de cette conférence montrent le caractère autoritaire qui aurait pu caractériser l'Allemagne impériale en cas de victoire militaire. Il serait d'ailleurs intéressant de voir si dans d'autres domaines de telles 'expérimentations' ont eu lieu en vue d'une éventuelle transposition en Allemagne après la guerre.

51. Insa Meinen relève aussi ce thème du 'pays infecté' pour la Deuxième Guerre mondiale: Meinen (2002, pp. 53-56).

52. Rapport du 19 janvier 1915 par un agent inspecteur; Pol14-18 - 420, AVB.

53. Les chiffres de comparaison sont issus de De Schaepdrijver (1983), de Corbin (1995, pp. 72-83) et de Le Naour (2002, pp. 165-172).

54. 'Liste der unter ärztlicher Aufsicht stehenden Personen'; Pol14-18 - 420, AVB.

55. Le nombre ' $n$ ' indique pour chaque cas d'analyse le nombre de prostituées pour lesquelles nous disposons de la donnée en question.

56. Verwaltungsbericht des Verwaltungschefs bei dem Generalgouverneur in Belgien für das Halbjahr Februar-April 1915; Rep. 89 H.-32456; Verwaltungsbericht des Verwaltungschefs bei dem Generalgouverneur in Belgien für die Zeit vom August-Okoter 1915, Rep. 89 H.-32457; GehStarch. PK; Verwaltungsbericht des Verwaltungschefs bei dem Generalgouverneur in Belgien für das Halbjahr Februar-April 1915; R1501-19455, BarchB; Halbjahresbericht für die Zeit vom 1. Februar bis 31. Juli 1917 - Gouvernement von Brüssel und Brabant; HS-2261, BayHStA.

57. De Schaepdrijver (1986, p. 555).

58. Pour la France au XIX ${ }^{\mathrm{e}}$ siècle, $c f$. Berlière (1992, pp. 40-41); pour la Belgique pendant la guerre cf. l'Echo de la presse du 5 janvier 1916; Pol14-18 - 420, AVB.

59. 33 listes de femmes arrêtées par le service des mœurs couvrant la période d'août 1915 à septembre 1918 sont conservées dans le dossier Pol14-18 - 417, AVB. Sur les réfugiés français en Belgique, $c f$. Delplancq (2001, pp. 71-80). 
60. Halasi (1917, p. 109).

61. Lettre du 21 juillet 1916 par Ostermann, employé de la Kommandantur à Bruxelles, à Crespin; Cp (sans numéro, dossier étiqueté 'colportage'), AVB et lettre du 21 septembre 1916 par Gerstein à Hurt, gouverneur militaire de Bruxelles; HS 2261, BayHStA.

62. Sans auteur, ni date; Pol14-18 - 420, AVB.

63. Les citations sont extraites de trois dossiers qui contiennent des rapports sur la prostitution à Bruxelles pendant la Première Guerre mondiale; Pol14-18 - 'colportage', Pol14-18 - 420, Pol14-18 - 500, AVB.

64. Lorenz (2000, pp. 115-121; 135-139).

65. La centrale de la dentellerie est une des nombreuses institutions mises en place par les autorités allemandes pour diriger l'économie belge.

66. La description se base sur les rapports conservés dans Pol14-18 - 446, AVB.

67. Barons du savon, sobriquet donné aux personnes qui ont profité de l'occupation allemande pour s'enrichir.

68. Procès-verbal du 19 juillet 1918 par la 4e division; Pol14-18 - 454, AVB; souligné par l'auteur.

69. Procès-verbal du 18 avril 1916 par le bureau des mœurs; Pol14-18 - 420, AVB.

70. Pour la Première Guerre mondiale en Allemagne, Liepmann (1930, pp. 79-130); pour la Deuxième Guerre mondiale en Belgique, Racine (1944).

71. « Niet wetende waarheen heb ik gedurende 3 weken gebedeld. 's nachts ging ik slapen in eene kar (...) Over acht dagen heb ik aan de Porte Louise, een ouden man ontmoet die mij gevraagd heeft met hem mede te gaan. Hij beloofde mij gelukkig te maken. (...) Hij heeft mij te eten gegeven en ik ben met hem blijven slapen in hetzelfde bed. Gedurende den nacht heeft hij mij willen gebruiken » (traduction par l'auteur), procès-verbal du 4 décembre 1917 par la 3e division; Pol14-18 - 465, AVB; nous n'avons pas changé l'orthographe de l'agent de police.

72. Sur les stratégies d'utilisation du système judiciaire pour régler des problèmes familiaux $c f$. De Koster (2001).

73. Procès-verbal du 18 septembre 1918 par le bureau des mœurs; Pol14-18 - 420, AVB; souligné par l'auteur.

74. Corbin (1995, p. 8).

75. Stadler, La faim et l'amour, sans date, extrait de Hirschfeld/Gaspar (1994, p. 325).

76. Bayet, Journal d'un Bruxellois pendant l'occupation allemande du 1er mai au 30 juin 1918 (manuscrit, p. 4156; M.L. 3546/21), Archives \& Musée de la Littérature à Bruxelles.

77. Procès-verbal du 17 juillet 1917 par la Division Centrale; Pol14-18 - 413, AVB.

78. Rapport du 15 mars 1918 par la 4e division; Cp 418, AVB et Fisher (s.d., pp. 179-186).

79. Notice du 31 mai 1915 par Gerstein; R1501-19374, BarchB.

80. «Mit dieser Organisation ist das Ziel erreicht, welches hier seit vielen Jahrzehnten erstrebt worden ist. ", extrait de Verwaltungsbericht des Verwaltungschefs bei dem Generalgouverneur in Belgien für das Halbjahr Februar-April 1915; Rep. 89 H.-32456, GehStarch PK.

81. Lettre du 17 mai 1915 par le ministre prussien de l'Intérieur au chancelier; R1501-19374, BarchB. Sur les administrations dans les territoires occupés comme lieux de promotion sociale $c f$. Föllmer (2000, p. 105).

82. «Der Dienst ist so eingerichtet, dass die deutschen Beamte die Verantwortung für die ordnungsgemässe Erledigung der Geschäfte tragen; für die Exekutive sind ihnen belgische Beamte überwiesen, mit welchen sie im Büro und im Revier zusammenarbeiten und über die sie während des Dienstes die Aufsicht ausüben. », extrait d'une lettre du 22 avril 1915 par Gerstein à von Sandt; R1501-19374, BarchB.

83. «Der Dienst in Brüssel wird zwar ausser von den deutschen Beamten zugleich von belgischen Schutzleuten versehen; aber für den Strassenaufsichtsdienst (dem wichtigsten Dienst der Sittenpolizei) müssen ihnen stets deutsche Beamte beigegeben werden, damit das deutsche 
Interesse gewahrt bleibt. ", extrait d'une lettre du 28 mars 1918 par Schaible au secrétaire d'État de l'Intérieur; R1501-19374, BarchB.

84. Rapport du 29 mai 1928 par le chef du bureau des mœurs; Pol14-18 - 420 et procès-verbal du 18 septembre 1918 du bureau des mœurs; Pol14-18 - 426, AVB; souligné par l'auteur.

85. Au printemps 1915, c'est-à-dire au début de la Sittenpolizei, dix-sept policiers belges y travaillent d'après le Berliner Tageblatt du 27 mars 1915; R1501-19376, BarchB.

86. Verwaltungsbericht des Verwaltungschefs bei dem Generalgouverneur in Belgien für das Halbjahr Februar-April 1915; Rep. 89 H.-32456, GehStarch PK.

87. Rapport du 29 août 1918 par Broché, chef du bureau des mœurs; Pol14-18 - 420, AVB.

88. Lettre du 10 décembre 1916 par Gerstein à l'administration communale de Bruxelles; Pol14-18 - 417, AVB.

89. Rapport du 9 décembre 1916 par Broché, chef du bureau des mœurs; Pol14-18 - 420, AVB.

90. Lettre du 4 mai 1915 par le collège échevinal de Saint-Josse-ten-Noode au chef de la police impériale; Pol14-18 - 420, rapport du 7 juillet 1915 par la 4e division et ordre $n^{\circ} 913$ du 11 mai 1915 par Lemonnier; Pol14-18 - 417, AVB.

91. Lettre du 21 septembre 1916 par Gerstein à Hurt; HS-2260, BayHStA.

92. Arrêté de condamnation du 21 avril 1915; Pol14-18 - 420, AVB.

93. Rapport du 4 janvier 1917 par Broché; Pol14-18 - 420, AVB.

94. Note du 6 juillet 1915 par Lemonnier; Pol14-18-417, AVB et Monjardet (1996, particulièrement les pp. 16-37).

95. Extrait de l'Écho de la presse du 5 janvier 1916; Pol14-18 - 420, AVB.

96. Nienhaus (1992, pp. 243-266) et les dossiers des inspectrices des mœurs et de la jeunesse; Personnel 1944, AVB.

97. E.a. Benn (1919). On peut cependant se demander si Benn y a vraiment travaillé et s'il ne s'agit pas d'une 'réalité littéraire'. En effet, sur les listes des médecins allemands qui sont payés par les autorités bruxelloises, son nom n'apparait jamais (cf. Pol14-18 - 417, AVB). Dans son autobiographie de 1921, on trouve le témoignage suivant «Ich war Arzt an einem Prostituiertenkrankenhaus, ein ganz isolierter Posten » Benn (1921, p. 57). Un autre écrivain s'est inspiré plus récemment de cet épisode pour écrire en roman sur le sujet: Mertens (1998, plus particulièrement pp. 87-166).

98. Annexe au budget du bureau de police et de l'Hôpital de Saint-Gilles pour le mois de septembre 1915; Pol14-18 - 417, AVB et Verwaltungsbericht des Verwaltungschefs bei dem Generalgouverneur in Belgien für das Halbjahr Februar-April 1915; Rep. 89 H.-32456, GehStarch PK.

99. von Drigalski utilise le terme de defekte Personcf. son rapport à la conférence du 8 octobre 1915; Massnahmen ... (s.d., p. 15).

100. Tous les chiffres de ce paragraphe proviennent des rapports suivants : Verwaltungsbericht des Verwaltungschefs bei dem Generalgouverneur in Belgien für das Halbjahr Februar-April 1915; Rep. 89 H.-32456, GehStarch PK; Halbjahresbericht für die Zeit vom 1. Februar bis 31. Juli 1917 - Gouvernement von Brüssel und Brabant et Halbjahresbericht der Provinz Brabant vom 1. Januar bis 30. Juni 1917 (Präsident der Zivilverwaltung für die Provinz Brabant); HS 2261, BayHStA; Verwaltungsbericht des Verwaltungschefs bei dem Generalgouverneur in Belgien für die Zeit vom Mai-Juli 1915; R1501-19455, BarchB. Les catégories sont reprises des rapports.

101. Rapport sanitaire (non daté) par le médecin militaire de la 6e armée couvrant les mois de février et mars; H(eeres)gr(uppe Kronprinz) Rupprecht - 59, BayHStA.

102. Gheude (1919, pp. 32-33). L'armée française hésite beaucoup plus entre une approche hygiéniste ou morale, la deuxième semblant l'emporter. Ainsi l'utilisation du préservatif est mal vue car elle risque de déculpabiliser les rapports sexuels. Le Naour (2002, pp. 194-198). 
103. Halbjahresbericht für die Zeit vom 1. Februar bis 31. Juli 1917 - Gouvernement von Brüssel und Brabant et rapport du 10 août 1915 par le médecin du Gouvernement général (nom inconnu); HS-2261, BayHStA.

104. La lecture parallèle des chapitres consacrés au péril vénérien chez Le Naour (2002, pp. 127-155) et Meinen (2002, pp. 37-49) montrent la pérennité de cette peur et ceci aussi bien en France qu'en Allemagne.

105. « die an dem Mark unserer Volkskraft nagen » (il n'y a pas de véritable traduction pour 'Volk' en français); Massnahmen..., (s.d., p. 5).

106. Pour une lecture novatrice du terme de démobilisation : Horne (2002).

107. Virgili (2000, pp. 230-234); depuis lors les travaux de Jean-Yves Le Naour ont relevé cette pratique en 1918 : Le Naour (2000).

108. Bayet, pp. 6345-6346 (voir note 77).

109. Rapport du 20 juillet 1917 par la 7e division; Pol14-18 - 510, AVB.

110. 'Avis du 4 mai 1915 concernant le traitement des dénonciations anonymes' par von Bissing, Un souvenir... (tome XXI, p. 34).

111. Nous nous basons sur le témoignage d'un consul honoraire (lettre du 24 novembre 1918 de celui-ci à Crespin) un rapport de police du 23 novembre 1918 par la Division Centrale et un procès-verbal du 26 novembre (!) 1918 par la 3e division; Pol14-18 - 439, AVB.

112. Lettre du 12 novembre 1918 par Holvoet à Lemonnier; $\mathrm{Cp} 420$ et la collection de procèsverbaux de la Conférence des Bourgmestres, CB 390, AVB.

113. Séance du 23 décembre 1921, Bulletin...1921 (1922, p. 2201).

114. Séance 19 mai 1924, Bulletin... 1924 (1925, p. 822).

115. Lettre anonyme du 13 mars 1919 à Max; Pol14-18 - 420, AVB.

116. Gubin (1995, p. 42).

117. Janssens (2001, p. 127).

118. Majerus (2003).

\section{RÉSUMÉS}

Pour les Allemands, Bruxelles devient pendant la Première Guerre mondiale un des symboles de la décadence de l'Etappe. Siège d'une importante administration occupante et lieu de passage pour de nombreux soldats, la capitale belge voit son nombre de prostituées fortement augmenté pendant les quatre années de l'occupation. Le monde prostitutionnel est profondément bouleversé. Pour les dirigeants allemands la prostitution est jugée d'un côté dangereuse pour la santé physique mais d'un autre côté nécessaire pour la santé psychique. L'approche pragmatique de l'administration allemande choque la société belge. Dans le contexte de l'occupation, la prostituée est considérée comme doublement traître sur le plan moral et sur le plan patriotique.

During the First World War, Brussels becomes one of the symbols of decadence of the Etappe. As the seat of an important German administration and as a resting point for numerous passing soldiers, the Belgian capital witnesses a sharp rise in the number of its prostitutes during the four years of occupation. The world of prostitution is turned upside down. The German authorities judge prostitution to be a danger to the physical health of the soldiers while at the same time they deem it necessary for their mental health. Indeed the pragmatic approach of the 
German administration shocks the Belgian population. In the context of the occupation, the prostitute is considered a double-traitor : morally and patriotically.

\section{AUTEUR}

\section{BENOIT MAJERUS}

CEGES, Résidence Palace/Block E, Rue de la Loi 155/Bte 2, B-1040 Bruxelles, Belgique, benoit.majerus@cegesoma.be Benoît Majerus (1975), licencié en histoire (ULB). Chercheur au Centre d'Études et de Documentation Guerre et Sociétés Contemporaines à Bruxelles, il travaille actuellement à l'élaboration d'une thèse de doctorat portant sur le maintien de l'ordre pendant les Première et Deuxième Guerres mondiales à Bruxelles. 